\title{
On Some New Generalized Difference Sequence Spaces of Nonabsolute Type
}

\author{
Osman Duyar, ${ }^{1}$ Serkan Demiriz, ${ }^{2}$ and Osman Özdemir ${ }^{2}$ \\ ${ }^{1}$ Anatolian High School, 60200 Tokat, Turkey \\ ${ }^{2}$ Department of Mathematics, Faculty of Arts and Science, Gaziosmanpaşa University, 60250 Tokat, Turkey
}

Correspondence should be addressed to Osman Duyar; osman-duyar@hotmail.com

Received 30 April 2014; Accepted 21 August 2014; Published 9 September 2014

Academic Editor: Tepper L. Gill

Copyright (c) 2014 Osman Duyar et al. This is an open access article distributed under the Creative Commons Attribution License, which permits unrestricted use, distribution, and reproduction in any medium, provided the original work is properly cited.

We define a new triangle matrix $\widehat{W}=\left(w_{n k}^{\lambda}\right)$ by the composition of the matrices $\Lambda=\left(\lambda_{n k}\right)$ and $B(r, s, t)$. Also, we introduce the sequence spaces $c_{0}^{\lambda}(\widehat{B}), c^{\lambda}(\widehat{B}), \ell_{\infty}^{\lambda}(\widehat{B})$, and $\ell_{p}^{\lambda}(\widehat{B})$ by using matrix domain of the matrix $\widehat{W}$ on the classical sequence spaces $c_{0}, c, \ell_{\infty}$, and $\ell_{p}$, respectively, where $1 \leq p<\infty$. Moreover, we show that the space $\mu^{\lambda}(\widehat{B})$ is norm isomorphic to $\mu$ for $\mu \in\left\{c_{0}, c, \ell_{\infty}, \ell_{p}\right\}$. Furthermore, we establish some inclusion relations concerning those spaces and determine $\alpha$-, $\beta$-, and $\gamma$-duals of those spaces and construct the Schauder bases $c_{0}^{\lambda}(\widehat{B}), c^{\lambda}(\widehat{B})$, and $\ell_{p}^{\lambda}(\widehat{B})$. Finally, we characterize the classes $\left(\mu_{1}^{\lambda}(\widehat{B}): \mu_{2}\right)$ of infinite matrices where $\mu_{1} \in\left\{c, c_{0}, \ell_{p}\right\}$ and $\mu_{2} \in\left\{\ell_{\infty}, c, c_{0}, \ell_{p}\right\}$.

\section{Preliminaries, Background, and Notation}

By a sequence space, we understand a linear subspace of the space $\omega$ of all complex sequences which contains $\phi$, the set of all finitely nonzero sequences. We write $\ell_{\infty}, c, c_{0}$, and $\ell_{p}$ for the classical sequence spaces of all bounded, convergent, null, and absolutely $p$-summable sequences, respectively, where $1 \leq p<\infty$. Also by $b s$ and $c s$, we denote the spaces of all bounded and convergent series, respectively. We assume throughout unless stated otherwise that $p, q>1$ with $p^{-1}+q^{-1}=1$ and use the convention that any term with negative subscript is equal to zero. We denote throughout that the collection of all finite subsets of $\mathbb{N}$ by $\mathscr{F}$.

Let $A=\left(a_{n k}\right)$ be an infinite matrix and $X, Y$ two sequence spaces. Then, $A$ defines a matrix mapping from $X$ to $Y$ and is denoted by $A: X \rightarrow Y$ if for every sequence $x=\left(x_{k}\right) \in X$ the sequence $A x=\left\{(A x)_{n}\right\}_{n \in \mathbb{N}}$, the $A$-transform of $x$, is in $Y$, where

$$
(A x)_{n}=\sum_{k} a_{n k} x_{k}, \quad(n \in \mathbb{N})
$$

By $(X: Y)$, denote the class of all matrices $A$ such that $A$ : $X \rightarrow Y$. Thus, $A \in(X: Y)$ if and only if the series on the right hand side of (1) converges for each $n \in \mathbb{N}$ and $x \in X$, and we have $A x=\left\{(A x)_{n}\right\}_{n \in \mathbb{N}} \in Y$ for all $x \in X$. A sequence $x \in \omega$ is said to be $A$-summable to $l$ if $A x$ converges to $l$, which is called the $A$-limit of $x$.

A matrix $A=\left(a_{n k}\right)$ is called a triangle if $a_{n k}=0$ for $k>n$ and $a_{n n} \neq 0$ for all $n, k \in \mathbb{N}$. It is trivial that $A(B x)=(A B) x$ holds for the triangle matrices $A, B$ and a sequence $x$. Further, a triangle matrix $U$ has a unique inverse $U^{-1}=V$ which is also a triangle matrix. Then, $x=U(V x)=V(U x)$ holds for all $x \in \omega$.

Let us give the definition of some triangle limitation matrices which are needed in the text. Let $q=\left(q_{k}\right)$ be a sequence of positive reals and write

$$
Q_{n}=\sum_{k=0}^{n} q_{k}, \quad(n \in \mathbb{N}) .
$$

Then the Cesàro mean of order one, Riesz mean with respect to the sequence $q=\left(q_{k}\right)$, and Euler mean of order $r$ with $0<$ 
$r<1$ are, respectively, defined by the matrices $C=\left(c_{n k}\right), R^{q}=$ $\left(r_{n k}^{q}\right)$, and $E^{r}=\left(e_{n k}^{r}\right)$, where

$$
\begin{gathered}
c_{n k}= \begin{cases}\frac{1}{n+1}, & (0 \leq k \leq n), \\
0, & (k>n),\end{cases} \\
r_{n k}^{q}= \begin{cases}\frac{q_{k}}{Q_{n}}, & (0 \leq k \leq n), \\
0, & (k>n),\end{cases} \\
e_{n k}^{r}= \begin{cases}\left(\begin{array}{l}
n \\
k
\end{array}\right)(1-r)^{n-k} r^{k}, & (0 \leq k \leq n), \\
0, & (k>n)\end{cases}
\end{gathered}
$$

for all $k, n \in \mathbb{N}$. We write $\mathcal{U}$ for the set of all sequences $u=$ $\left(u_{k}\right)$ such that $u_{k} \neq 0$ for all $k \in \mathbb{N}$. For $u \in \mathscr{U}$, let $1 / u=$ $\left(1 / u_{k}\right)$. Let $z, u, v \in \mathcal{U}$, and define the summation matrix $S=$ $\left(s_{n k}\right)$, the difference matrix $\Delta=\left(\Delta_{n k}^{(1)}\right)$, and the generalized weighted mean or factorable matrix $G(u, v)=\left(g_{n k}\right), A_{u}^{r}=$ $\left\{a_{n k}^{r}(u)\right\}, \Delta^{(m)}=\left(\Delta_{n k}^{(m)}\right)$ by

$$
\begin{gathered}
s_{n k}= \begin{cases}1, & (0 \leq k \leq n), \\
0, & (k>n),\end{cases} \\
\Delta_{n k}^{(1)}= \begin{cases}(-1)^{n-k}, & (n-1 \leq k \leq n), \\
0, & (0 \leq k<n-1 \text { or } k>n),\end{cases} \\
g_{n k}= \begin{cases}u_{n} v_{k}, & (0 \leq k \leq n), \\
0, & (k>n),\end{cases} \\
a_{n k}^{r}(u)= \begin{cases}\frac{1+r^{k}}{n+1} u_{k}, & (0 \leq k \leq n), \\
0, & (k>n),\end{cases} \\
\Delta_{n k}^{(m)}= \begin{cases}(-1)^{n-k}\left(\begin{array}{cc}
m \\
n-k
\end{array}\right), & (\max \{0, n-m\} \leq k \leq n), \\
0, & (0 \leq k<n-1 \text { or } k>n)\end{cases}
\end{gathered}
$$

for all $k, n \in \mathbb{N}$, where $u_{n}$ and $v_{k}$ depend only on $n$ and $k$, respectively. Let $r$ and $s$ be nonzero real numbers, and define the generalized difference matrix $B(r, s)=\left\{b_{n k}(r, s)\right\}$ by

$$
b_{n k}(r, s)= \begin{cases}r, & (k=n), \\ s, & (k=n-1), \\ 0, & (0 \leq k<n-1 \text { or } k>n),\end{cases}
$$

for all $k, n \in \mathbb{N}$. We note that if we choose $r=1$ and $s=-1$ then the matrix $B(r, s)$ is reduced to the backward difference.

For a sequence space $X$, the matrix domain $X_{A}$ of an infinite matrix $A$ in the space $X$ is defined by

$$
X_{A}=\left\{x=\left(x_{k}\right) \in \omega: A x \in X\right\},
$$

which is a sequence space. If $A$ is triangle, then one can easily observe that the sequence spaces $X_{A}$ and $X$ are linearly isomorphic; that is, $X_{A} \cong X$.
Following Başar [1, page 51], we note that although in the most cases the new sequence space $X_{A}$ generated by the limitation matrix $A$ from a sequence space $X$ is the expansion or the contraction of the original space $X$, it may be observed in some cases that those spaces overlap. Indeed, one can easily see that the inclusion, $X_{S} \subset X$, strictly holds for $X \in\left\{\ell_{\infty}, c, c_{0}\right\}$. As this, one can deduce that the inclusion $X \subset X_{\Delta^{(1)}}$ also strictly holds for $X \in\left\{\ell_{\infty}, c, c_{0}, \ell_{p}\right\}$. However, if we define $X=c_{0} \oplus \operatorname{span}\{z\}$ with $z=((-1))^{k}$, that is, $x \in X$ if and only if $x:=\beta+\alpha z$ for some $\beta \in c_{0}$ and some $\alpha \in \mathbb{C}$, and consider the matrix $A$ with the rows $A_{n}$ defined by $A_{n}=(-1)^{n} e^{(n)}$ for all $n \in \mathbb{N}$, we have $A e=z \in X$ but $A z=e \notin X$ which lead us to the consequences that $z \in X \backslash X_{A}$ and $e \in X_{A} \backslash X$, where $e=(1,1,1, \ldots)$ and $e^{(n)}$ is a sequence whose only nonzero term is a 1 in $n$th place for each $n \in \mathbb{N}$. That is to say, the sequence spaces $X_{A}$ and $X$ overlap but neither contains the other.

The approach constructing a new sequence space by means of the matrix domain of a particular limitation method has recently been employed by Wang [2], $\mathrm{Ng}$ and Lee [3], Malkowsky [4], Altay and Başar [5], Malkowsky and Savaş [6], Başarır [7], Aydın and Başar [8], Başar et al. [9], Şengönül and Başar [10], Altay [11], Polat and Başar [12], and Malkowsky et al. [13]. $\Delta, \Delta^{2}$ and $\Delta^{m}$ are the transposes of the matrices $\Delta^{(1)}, \Delta^{(2)}$, and $\Delta^{(m)}$, respectively, and $c_{0}(u, p)$ are the spaces consisting of the sequences $x=\left(x_{k}\right)$ such that $u x=\left(u_{k} x_{k}\right)$ in the spaces $c_{0}(p)$ and $c(p)$ for $u \in$ $\mathcal{U}$, respectively, studied by Başarır [7]. More recently, the generalized difference matrix $B(r, s)=\left\{b_{n k}(r, s)\right\}$ has been used by Kirişçi and Başar [14] for generalizing the difference spaces $\ell_{\infty}(\Delta), c(\Delta), c_{0}(\Delta)$, and $b v_{p}$. Finally, the new technique for deducing certain topological properties, for example, $A B$-, $K B$-, and $A D$-properties, and determining the $\beta$ - and $\gamma$-duals of the domain of a triangle matrix in a sequence space has been given by Altay and Başar [15].

Let $r, s$, and $t$ be nonzero real numbers, and define the generalized difference matrix $\widehat{B}=B(r, s, t)=\left\{b_{n k}(r, s, t)\right\}$ by

$$
b_{n k}(r, s, t)= \begin{cases}r, & (k=n), \\ s, & (k=n-1) \\ t, & (k=n-2), \\ 0, & (0 \leq k<n-1 \text { or } k>n)\end{cases}
$$

for all $n, k \in \mathbb{N}$. The inverse $B^{-1}(r, s, t)=\left\{d_{n k}(r, s, t)\right\}$ of $B(r, s, t)=\left\{b_{n k}(r, s, t)\right\}$ is given by

$$
\begin{aligned}
& d_{n k}(r, s, t) \\
& = \begin{cases}\frac{1}{r} \sum_{v=0}^{n-k}\left(\frac{-s+\sqrt{s^{2}-4 t r}}{2 r}\right)^{n-k-v} \\
\quad \times\left(\frac{-s-\sqrt{s^{2}-4 t r}}{2 r}\right)^{v}, & (0 \leq k \leq n), \\
0, & (k>n) .\end{cases}
\end{aligned}
$$

We should record here that $B(r, s, 0)=B(r, s), B(1,-2,1)=$ $\Delta^{(2)}$, and $B(1,-1,0)=\Delta^{(1)}$. So, the results related to the matrix domain of the triple band matrix $B(r, s, t)$ are more 
general and more comprehensive than the consequences on the matrix domain of $B(r, s), \Delta^{(2)}$ and $\Delta^{(1)}$ and include them. We assume throughout that $\lambda=\left(\lambda_{k}\right)_{k=0}^{\infty}$ is a strictly increasing sequence of positive reals tending to $\infty$; that is

$$
0<\lambda_{0}<\lambda_{1}<\cdots, \quad \lim _{k \rightarrow \infty} \lambda_{k}=\infty
$$

The main purpose of the present paper is to introduce the sequence spaces $\mu^{\lambda}(\widehat{B})$ and to determine the $\alpha$-, $\beta$-, and $\gamma$-duals of this space, where $\mu$ denotes throughout any of the classical spaces $\ell_{\infty}, c, c_{0}$, or $\ell_{p}$, and $\widehat{B}$ is the triple band matrix $B(r, s, t)$ and the sequence $\lambda=\left(\lambda_{k}\right)$ is defined in (9). Furthermore, the Schauder bases for the spaces $c_{0}^{\lambda}(\widehat{B}), c^{\lambda}(\widehat{B})$ and $\ell_{p}^{\lambda}(\widehat{B})$ are given, and some topological properties of the spaces $c_{0}^{\lambda}(\widehat{B}), c^{\lambda}(\widehat{B})$, and $\ell_{p}^{\lambda}(\widehat{B})$ are examined. Finally, some classes of matrix mappings on the spaces $\mu^{\lambda}(\widehat{B})$ are characterized.

The paper is organized as follows. In Section 2, the $B K$-spaces $c_{0}^{\lambda}(\widehat{B}), c^{\lambda}(\widehat{B}), \ell_{\infty}^{\lambda}(\widehat{B})$, and $\ell_{p}^{\lambda}(\widehat{B})$ of generalized difference sequence spaces are introduced and the Schauder bases of the spaces $c_{0}^{\lambda}(\widehat{B}), c^{\lambda}(\widehat{B})$, and $\ell_{p}^{\lambda}(\widehat{B})$ are given. In Section 3, some inclusion relations concerning these spaces are examined. In Section 4 , the $\alpha$-, $\beta$-, and $\gamma$-duals of the generalized difference sequence spaces $\mu^{\lambda}(\widehat{B})$ of nonabsolute type are determined. In Section 5 , the classes $\left(\mu_{1}^{\lambda}(\widehat{B}): \mu_{2}\right)$ of infinite matrices are characterized, where $\mu_{1} \in\left\{c, c_{0}, \ell_{p}\right\}$ and $\mu_{2} \in\left\{\ell_{\infty}, c, c_{0}, \ell_{p}\right\}$.

\section{The Difference Sequence Spaces $\mu^{\lambda}(\widehat{B})$ of Nonabsolute Type}

The difference sequence spaces have been studied by several authors in different ways [16-34]. In the present section, we introduce the spaces $c_{0}^{\lambda}(\widehat{B}), c^{\lambda}(\widehat{B}), \ell_{\infty}^{\lambda}(\widehat{B})$, and $\ell_{p}^{\lambda}(\widehat{B})$ and show that these spaces are $B K$-spaces of nonabsolute type which are norm isomorphic to the spaces $c_{0}, c, \ell_{\infty}$, and $\ell_{p}$, respectively. Furthermore, we give the bases of the spaces $c_{0}^{\lambda}(\widehat{B}), c^{\lambda}(\widehat{B})$, and $\ell_{p}^{\lambda}(\widehat{B})$.

We say that a sequence $x=\left(x_{k}\right) \in \omega$ is $\lambda$-convergent to the number $l \in \mathbb{C}$, called the $\lambda$-limit of $x$, if $(\Lambda x)_{n} \rightarrow l$ as $n \rightarrow \infty$ where

$$
(\Lambda x)_{n}=\frac{1}{\lambda_{n}} \sum_{k=0}^{n}\left(\lambda_{k}-\lambda_{k-1}\right) x_{k} ; \quad(n \in \mathbb{N})
$$

In particular, we say that $x$ is a $\lambda$-null sequence if $(\Lambda x)_{n} \rightarrow$ 0 as $n \rightarrow \infty$. Further, we say that $x$ is $\lambda$-bounded if $\sup _{n \in \mathbb{N}}\left|(\Lambda x)_{n}\right|<\infty$, [35]. Recently, Mursaleen and Noman
$[35,36]$ studied the sequence spaces $c_{0}^{\lambda}, c^{\lambda}, \ell_{\infty}^{\lambda}$, and $\ell_{p}^{\lambda}$ of nonabsolute type as follows:

$$
\begin{aligned}
& c_{0}^{\lambda}=\left\{x=\left(x_{k}\right) \in \omega: \lim _{n \rightarrow \infty} \frac{1}{\lambda_{n}} \sum_{k=0}^{n}\left(\lambda_{k}-\lambda_{k-1}\right) x_{k}=0\right\}, \\
& c^{\lambda}=\left\{x=\left(x_{k}\right) \in \omega: \lim _{n \rightarrow \infty} \frac{1}{\lambda_{n}} \sum_{k=0}^{n}\left(\lambda_{k}-\lambda_{k-1}\right) x_{k} \text { exists }\right\}, \\
& \ell_{\infty}^{\lambda}=\left\{x=\left(x_{k}\right) \in \omega: \sup _{n \in \mathbb{N}}\left|\frac{1}{\lambda_{n}} \sum_{k=0}^{n}\left(\lambda_{k}-\lambda_{k-1}\right) x_{k}\right|<\infty\right\}, \\
& \ell_{p}^{\lambda}=\left\{x=\left(x_{k}\right) \in \omega: \sum_{n=0}^{\infty}\left|\frac{1}{\lambda_{n}} \sum_{k=0}^{n}\left(\lambda_{k}-\lambda_{k-1}\right) x_{k}\right|<\infty\right\} .
\end{aligned}
$$

On the other hand, we define the matrix $\Lambda=\left(\lambda_{n k}\right)$ for all $n, k \in \mathbb{N}$ by

$$
\lambda_{n k}= \begin{cases}\frac{\lambda_{k}-\lambda_{k-1}}{\lambda_{n}}, & (0 \leq k \leq n) \\ 0, & (k>n) .\end{cases}
$$

Then, the space $\mu^{\lambda}$ can be restated with the notation of (6) that

$$
c_{0}^{\lambda}=\left\{c_{0}\right\}_{\Lambda}, \quad c^{\lambda}=c_{\Lambda}, \quad \ell_{\infty}^{\lambda}=\left\{\ell_{\infty}\right\}_{\Lambda}, \quad \ell_{p}^{\lambda}=\left\{\ell_{p}\right\}_{\Lambda} .
$$

More recently, Sönmez [37] has defined the sequence spaces $\ell_{\infty}(\widehat{B}), c(\widehat{B}), c_{0}(\widehat{B})$, and $\ell_{p}(\widehat{B})$ as follows:

$$
\begin{aligned}
& \ell_{\infty}(\widehat{B})=\left\{x=\left(x_{k}\right) \in \omega: \sup _{k \in \mathbb{N}}\left|r x_{k}+s x_{k-1}+t x_{k-2}\right|<\infty\right\}, \\
& c(\widehat{B})=\left\{x=\left(x_{k}\right) \in \omega:\right. \\
&\left.\exists l \in \mathbb{C} \ni \lim _{k \rightarrow \infty}\left|r x_{k}+s x_{k-1}+t x_{k-2}-l\right|=0\right\}, \\
& \mathcal{c}_{0}(\widehat{B})=\left\{x=\left(x_{k}\right) \in \omega: \lim _{k \rightarrow \infty}\left|r x_{k}+s x_{k-1}+t x_{k-2}\right|=0\right\}, \\
& \ell_{p}(\widehat{B})=\left\{x=\left(x_{k}\right) \in \omega: \sum_{k}\left|r x_{k}+s x_{k-1}+t x_{k-2}\right|^{p}<\infty\right\} .
\end{aligned}
$$

In fact, the sequence spaces $\ell_{\infty}(\widehat{B}), c(\widehat{B}), c_{0}(\widehat{B})$, and $\ell_{p}(\widehat{B})$ can be considered as the set of all sequences whose $B(r, s, t)$ transforms are in the spaces $\ell_{\infty}, c, c_{0}$, and $\ell_{p}$, respectively. That is,

$$
\begin{array}{cc}
\ell_{\infty}(\widehat{B})=\left\{\ell_{\infty}\right\}_{B(r, s, t)}, & c(\widehat{B})=c_{B(r, s, t)}, \\
c_{0}(\widehat{B})=\left\{c_{0}\right\}_{B(r, s, t)}, \quad \ell_{p}(\widehat{B})=\left\{\ell_{p}\right\}_{B(r, s, t)} .
\end{array}
$$


Now, we introduce the difference sequence spaces $\ell_{\infty}^{\lambda}(\widehat{B}), c^{\lambda}(\widehat{B}), c_{0}^{\lambda}(\widehat{B})$, and $\ell_{p}^{\lambda}(\widehat{B})$ as follows:

$$
\begin{aligned}
& \ell_{\infty}^{\lambda}(\widehat{B})=\left\{x=\left(x_{n}\right) \in \omega:\right. \\
& \sup _{n \in \mathbb{N}} \mid \frac{1}{\lambda_{n}} \sum_{k=0}^{n}\left(\lambda_{k}-\lambda_{k-1}\right) \\
&\left.\quad \times\left(r x_{k}+s x_{k-1}+t x_{k-2}\right) \mid<\infty\right\},
\end{aligned}
$$$$
c^{\lambda}(\widehat{B})=\left\{x=\left(x_{n}\right) \in \omega:\right.
$$$$
\lim _{n \rightarrow \infty} \frac{1}{\lambda_{n}} \sum_{k=0}^{n}\left(\lambda_{k}-\lambda_{k-1}\right)
$$$$
\left.\times\left(r x_{k}+s x_{k-1}+t x_{k-2}\right) \text { exists }\right\},
$$$$
c_{0}^{\lambda}(\widehat{B})=\left\{x=\left(x_{n}\right) \in \omega:\right.
$$$$
\lim _{n \rightarrow \infty} \frac{1}{\lambda_{n}} \sum_{k=0}^{n}\left(\lambda_{k}-\lambda_{k-1}\right)
$$$$
\left.\times\left(r x_{k}+s x_{k-1}+t x_{k-2}\right)=0\right\},
$$

$$
\begin{aligned}
\ell_{p}^{\lambda}(\widehat{B})=\left\{x=\left(x_{n}\right) \in \omega:\right. & \\
& \sum_{n} \mid \frac{1}{\lambda_{n}} \sum_{k=0}^{n}\left(\lambda_{k}-\lambda_{k-1}\right) \\
& \left.\times\left.\left(r x_{k}+s x_{k-1}+t x_{k-2}\right)\right|^{p}<\infty\right\} .
\end{aligned}
$$

Now, we define the triangle matrix $\widehat{W}=\left(w_{n k}^{\lambda}\right)=\Lambda \widehat{B}$; that is,

$$
w_{n k}^{\lambda}= \begin{cases}\frac{\left.r\left(\lambda_{n-1}-\lambda_{n-2}\right)+s\left(\lambda_{n-1}\right)+\lambda_{n-1}\right)}{\lambda_{n}}, & (k=n-1), \\ \left.+t\left(\lambda_{k+2}-\lambda_{k+1}\right)\right) \times\left(\lambda_{n}\right)^{-1}, & (k<n-1), \\ \frac{r\left(\lambda_{n}-\lambda_{n-1}\right)}{\lambda_{n}}, & (k=n), \\ 0, & \text { (otherwise) }\end{cases}
$$

for all $k, n \in \mathbb{N}$. Further, for any sequence $x=\left(x_{k}\right)$ we define the sequence $y=\left\{y_{k}(\lambda)\right\}$ which will be used, as the $\widehat{W}$-transform of $x$; that is,

$$
\begin{aligned}
y_{k}(\lambda)= & \sum_{j=0}^{k-2} \frac{r\left(\lambda_{j}-\lambda_{j-1}\right)+s\left(\lambda_{j+1}-\lambda_{j}\right)+t\left(\lambda_{j+2}-\lambda_{j+1}\right)}{\lambda_{k}} x_{j} \\
& +\frac{r\left(\lambda_{k-1}-\lambda_{k-2}\right)+s\left(\lambda_{k}-\lambda_{k-1}\right)}{\lambda_{k}} x_{k-1} \\
& +\frac{r\left(\lambda_{k}-\lambda_{k-1}\right)}{\lambda_{k}} x_{k} ; \quad(k \in \mathbb{N}) .
\end{aligned}
$$

In fact, the sequence spaces $c_{0}^{\lambda}(\widehat{B}), c^{\lambda}(\widehat{B}), \ell_{\infty}^{\lambda}(\widehat{B})$, and $\ell_{p}^{\lambda}(\widehat{B})$ can be considered as the set of all sequences whose $\widehat{W}$ transforms are in the spaces $c_{0}, c, \ell_{\infty}$, and $\ell_{p}$, respectively. That is,

$$
\begin{array}{cc}
c_{0}^{\lambda}(\widehat{B})=\left\{c_{0}\right\}_{\widehat{W}}, & c^{\lambda}(\widehat{B})=c_{\widehat{W}}, \\
\ell_{\infty}^{\lambda}(\widehat{B})=\left\{\ell_{\infty}\right\}_{\widehat{W}}, & \ell_{p}^{\lambda}(\widehat{B})=\left\{\ell_{p}\right\}_{\widehat{W}} .
\end{array}
$$

Since the proof may also be obtained in a similar way as the other spaces, to avoid the repetition of the similar statements, we give the proof only for one of those spaces. Now, we may begin with the following theorem which is essential in the study.

Theorem 1. (i) The difference sequence spaces $c_{0}^{\lambda}(\widehat{B}), c^{\lambda}(\widehat{B})$, and $\ell_{\infty}^{\lambda}(\widehat{B})$ are BK-spaces with the norm $\|x\|_{c_{0}^{\lambda}(\widehat{B})}=\|x\|_{\mathcal{C}^{\lambda}(\widehat{B})}=$ $\|x\|_{\ell_{\infty}^{\lambda}(\widehat{B})}=\|\widehat{W} x\|_{\infty}$; that is,

$$
\|x\|_{\mathcal{C}_{0}^{\lambda}(\widehat{B})}=\|x\|_{\mathcal{C}^{\lambda}(\widehat{B})}=\|x\|_{\ell_{\infty}^{\lambda}(\widehat{B})}=\sup _{n \in \mathbb{N}}\left|(\widehat{W} x)_{n}\right| .
$$

(ii) Let $1 \leq p<\infty$. Then $\ell_{p}^{\lambda}(\widehat{B})$ is a BK-space with the norm $\|x\|_{\ell_{p}^{\lambda}(\widehat{B})}=\|\widehat{W} x\|_{\ell_{p}}$; that is,

$$
\|x\|_{\ell_{p}^{\lambda}(\widehat{B})}=\left(\sum_{n}\left|(\widehat{W} x)_{n}\right|^{p}\right)^{1 / p} .
$$

Proof. Since (19) holds and $c_{0}, c, \ell_{\infty}$, and $\ell_{p}$ are $B K$-spaces with respect to their natural norms (see [1, pages 16-17]) and the matrix $\widehat{W}$ is a triangle, Theorem 4.3 .12 of Wilansky [38, page 63] gives the fact that $c_{0}^{\lambda}(\widehat{B}), c^{\lambda}(\widehat{B}), \ell_{\infty}^{\lambda}(\widehat{B})$, and $\ell_{p}^{\lambda}(\widehat{B})$ are $B K$-spaces with the given norms. This completes the proof.

Remark 2. Let $|x|=\left(\left|x_{k}\right|\right)$. Then the absolute property does not hold on the space $\mu^{\lambda}(\widehat{B})$; that is, $\|x\|_{\mu^{\lambda}(\widehat{B})} \neq\||x|\|_{\mu^{\lambda}(\widehat{B})}$. This can be shown for at least one sequence in those spaces. Hence $\mu^{\lambda}(\widehat{B})$ is the sequence space of nonabsolute type.

Theorem 3. The sequence spaces $c_{0}^{\lambda}(\widehat{B}), c^{\lambda}(\widehat{B}), \ell_{\infty}^{\lambda}(\widehat{B})$, and $\ell_{p}^{\lambda}(\widehat{B})$ of nonabsolute type are norm isomorphic to the spaces $c_{0}, c, \ell_{\infty}$, and $\ell_{p}$, respectively; that is, $c_{0}^{\lambda}(\widehat{B}) \cong c_{0}, c^{\lambda}(\widehat{B}) \cong c$, $\ell_{\infty}^{\lambda}(\widehat{B}) \cong \ell_{\infty}$, and $\ell_{p}^{\lambda}(\widehat{B}) \cong \ell_{p}$. 
Proof. We prove the theorem for the space $c_{0}^{\lambda}(\widehat{B})$. To prove our assertion we should show the existence of a linear bijection between the spaces $c_{0}^{\lambda}(\widehat{B})$ and $c_{0}$. Let $T: c_{0}^{\lambda}(\widehat{B}) \rightarrow c_{0}$ be defined by (18). Then, $T x=y=\widehat{W} x \in c_{0}$ for every $x \in c_{0}^{\lambda}(\widehat{B})$ and the linearity of $T$ is clear. Further, it is trivial that $x=\theta$ whenever $T x=\theta$ and hence $T$ is injective.

Moreover, let $y=\left(y_{k}\right) \in c_{0}$ and we define the sequence $x=\left\{x_{k}(\lambda)\right\}$ by

$$
\begin{gathered}
x_{k}(\lambda)=\sum_{j=0}^{k} \frac{1}{r} \sum_{v=0}^{k-j}\left(\frac{-s+\sqrt{s^{2}-4 t r}}{2 r}\right)^{k-j-v}\left(\frac{-s-\sqrt{s^{2}-4 t r}}{2 r}\right)^{v} \\
\times \sum_{i=j-1}^{j}(-1)^{j-i} \frac{\lambda_{i}}{\lambda_{j}-\lambda_{j-1}} y_{i} .
\end{gathered}
$$

Then we obtain

$$
\begin{aligned}
& r x_{k}(\lambda)+s x_{k-1}(\lambda)+t x_{k-2}(\lambda) \\
& \quad=\sum_{j=k-1}^{k}(-1)^{k-j} \frac{\lambda_{j}}{\lambda_{k}-\lambda_{k-1}} y_{j}, \quad \forall k \in \mathbb{N} .
\end{aligned}
$$

Hence, we get for every $n \in \mathbb{N}$ that

$$
\begin{aligned}
(\widehat{W} x)_{n} & =\frac{1}{\lambda_{n}} \sum_{k=0}^{n}\left(\lambda_{k}-\lambda_{k-1}\right)\left(r x_{k}+s x_{k-1}+t x_{k-2}\right) \\
& =\frac{1}{\lambda_{n}} \sum_{k=0}^{n} \sum_{j=k-1}^{k}(-1)^{k-j} \lambda_{j} y_{j}=y_{n} .
\end{aligned}
$$

This shows that $\widehat{W} x=y$ and since $y \in c_{0}$, we conclude that $\widehat{W} x \in c_{0}$. Thus, we deduce that $x \in c_{0}^{\lambda}(\widehat{B})$ and $T x=y$. Hence $T$ is surjective.

Moreover one can easily see for every $x \in c_{0}^{\lambda}(\widehat{B})$ that

$$
\|T x\|_{\infty}=\|y\|_{\infty}=\|\widehat{W} x\|_{\infty}=\|x\|_{c_{0}^{\lambda}(\widehat{B})}
$$

which means that $T$ is norm preserving. Consequently $T$ is a linear bijection which shows that the spaces $c_{0}^{\lambda}(\widehat{B})$ and $c_{0}$ are linearly isomorphic, as desired.

Let $(X,\|\cdot\|)$ be a normed space. A sequence $\left(b_{k}\right)$ of points of $X$ is called a Schauder basis for $X$ if and only if, for each $x \in X$, there exists a unique sequence $\left(\alpha_{k}\right)$ of scalars such that $x=\sum_{k} \alpha_{k} b_{k}$; that is,

$$
\lim _{n \rightarrow \infty}\left\|x-\sum_{k=0}^{n} \alpha_{k} b_{k}\right\|=0
$$

Because of the isomorphism $T$, which is defined in the proof of Theorem 3, the inverse image of the basis of the spaces $c_{0}, c$, and $\ell_{p}$ are the basis of new spaces $c_{0}^{\lambda}(\widehat{B}), c^{\lambda}(\widehat{B})$, and $\ell_{p}^{\lambda}(\widehat{B})$, respectively. Therefore, we have the following.
Theorem 4. Let $\alpha_{k}(\lambda)=(\widehat{W} x)_{k}$ for all $k \in \mathbb{N}$ and $\lim _{k \rightarrow \infty}(\widehat{W} x)_{k}=l$. Define the sequence $b^{(k)}(\lambda)=\left\{b_{n}^{(k)}(\lambda)\right\}_{n \in \mathbb{N}}$ for every fixed $k \in \mathbb{N}$ by

$$
b_{n}^{(k)}(\lambda)= \begin{cases}\frac{d_{n k}(r, s, t) \lambda_{k}}{\lambda_{k}-\lambda_{k-1}}-\frac{d_{n, k+1}(r, s, t) \lambda_{k}}{\lambda_{k+1}-\lambda_{k}}, & (n>k) \\ \frac{1}{r} \frac{\lambda_{k}}{\left(\lambda_{k}-\lambda_{k-1}\right)}, & (n=k) \\ 0, & (n<k) .\end{cases}
$$

Then, the following statements hold.

(i) The sequence $\left\{b^{(k)}(\lambda)\right\}_{k \in \mathbb{N}}$ is a basis for the spaces $c_{0}^{\lambda}(\widehat{B})$ and $\ell_{p}^{\lambda}(\widehat{B})$; any $x \in c_{0}^{\lambda}(\widehat{B})$ or $x \in \ell_{p}^{\lambda}(\widehat{B})$ has a unique representation of the form $x=\sum_{k} \alpha_{k}(\lambda) b^{(k)}(\lambda)$.

(ii) The sequence $\left\{b, b^{(0)}(\lambda), b^{(1)}(\lambda), b^{(2)}(\lambda), \ldots\right\}$ is a basis for the space $c^{\lambda}(\widehat{B})$ and any $x \in c^{\lambda}(\widehat{B})$ has a unique representation of the form $x=l b+\sum_{k}\left[\alpha_{k}(\lambda)-l\right] b^{(k)}(\lambda)$, where $b=\left(b_{k}\right)=\left(\sum_{j=0}^{k} d_{k j}\right)$.

\section{The Inclusion Relations}

In the present section, we prove some inclusion relations concerning the spaces $c_{0}^{\lambda}(\widehat{B}), c^{\lambda}(\widehat{B}), \ell_{\infty}^{\lambda}(\widehat{B})$, and $\ell_{p}^{\lambda}(\widehat{B})$.

Theorem 5. The inclusion $c_{0}^{\lambda}(\widehat{B}) \subset c^{\lambda}(\widehat{B})$ strictly holds.

Proof. It is obvious that the inclusion $c_{0}^{\lambda}(\widehat{B}) \subset c^{\lambda}(\widehat{B})$ holds. Further to show that this inclusion is strict, consider the sequence $x=\left(x_{k}\right)$ defined by

$$
x_{k}=\sum_{j=0}^{k} d_{k j}
$$

for all $k \in \mathbb{N}$. Then

$$
r x_{k}+s x_{k-1}+t x_{k-2}=\sum_{j=k-1}^{k}(-1)^{k-j} \frac{\lambda_{j}}{\lambda_{k}-\lambda_{k-1}} .
$$

Then one can easily see that $\widehat{W} x=e \in c \backslash c_{0}$, where $e=$ $(1,1,1, \ldots)$. Thus, this sequence $x$ is in $c^{\lambda}(\widehat{B})$ but not in $c_{0}^{\lambda}(\widehat{B})$. Hence, the inclusion $c_{0}^{\lambda}(\widehat{B}) \subset c^{\lambda}(\widehat{B})$ is strict and this completes the proof of the theorem.

Theorem 6. If $r+s+t=0$ then the inclusion $c \subset c_{0}^{\lambda}(\widehat{B})$ strictly holds.

Proof. Suppose that $r+s+t=0$ and $x \in c$. Then $\widehat{B} x=$ $\left(r x_{k}+s x_{k-1}+t x_{k-2}\right) \in c_{0}$ and hence $\widehat{B} x \in c_{0}^{\lambda}$ since the inclusion $c_{0} \subset c_{0}^{\lambda}$ holds [35]. This shows that $x \in c_{0}^{\lambda}(\widehat{B})$; that is, $c \subset$ $c_{0}^{\lambda}(\widehat{B})$. Further consider the sequence $y=\left(y_{k}\right)$ defined by $y_{k}=$ $\ln (k+3)$ for all $k \in \mathbb{N}$. Then it is trivial that $y \notin c$. On the other hand, it can easily be seen that $\widehat{B} y \in c_{0}$. Hence, $y \in$ $c_{0}^{\lambda}(\widehat{B})$. Thus the sequence $y \in c_{0}^{\lambda}(\widehat{B}) \backslash c$. Hence, the inclusion $c \subset c_{0}^{\lambda}(\widehat{B})$ is strict. This completes the proof. 
Lemma 7. $A \in\left(\ell_{\infty}: c_{0}\right)$ if and only if $\lim _{n \rightarrow \infty} \sum_{k}\left|a_{n k}\right|=0$.

Theorem 8. Let $z=\left(z_{k}\right)$ be defined by

$$
z_{k}=\left|\frac{r\left(\lambda_{k}-\lambda_{k-1}\right)+s\left(\lambda_{k+1}-\lambda_{k}\right)+t\left(\lambda_{k+2}-\lambda_{k+1}\right)}{\lambda_{k}-\lambda_{k-1}}\right|
$$

for all $k \in \mathbb{N}$. Then the inclusion $\ell_{\infty} \subset c_{0}^{\lambda}(\widehat{B})$ strictly holds if and only if $z \in c_{0}^{\lambda}$.

Proof. Let $\ell_{\infty}$ be a subset of $c_{0}^{\lambda}(\widehat{B})$. Then we obtain that $\widehat{W} x \in$ $c_{0}$ for every $x \in \ell_{\infty}$ and the matrix $\widehat{W}=\left(w_{n k}^{\lambda}\right)$ is in the class $\left(\ell_{\infty}: c_{0}\right)$. By using Lemma 7 it follows that

$$
\lim _{n \rightarrow \infty} \sum_{k}\left|w_{n k}^{\lambda}\right|=0
$$

Now, by taking into account the definition of matrix $\widehat{W}=$ $\left(w_{n k}^{\lambda}\right)$ given by (17), we have for every $n \in \mathbb{N}$ that

$$
\begin{aligned}
& \sum_{k}\left|w_{n k}^{\lambda}\right| \\
& =\frac{1}{\lambda_{n}} \sum_{k=0}^{n-2}\left|r\left(\lambda_{k}-\lambda_{k-1}\right)+s\left(\lambda_{k+1}-\lambda_{k}\right)+t\left(\lambda_{k+2}-\lambda_{k+1}\right)\right| \\
& \quad+\left|\frac{r\left(\lambda_{n-1}-\lambda_{n-2}\right)+s\left(\lambda_{n}-\lambda_{n-1}\right)}{\lambda_{n}}\right|+|r| \frac{\left(\lambda_{n}-\lambda_{n-1}\right)}{\lambda_{n}} .
\end{aligned}
$$

By Lemma 7, we have that

$$
\begin{gathered}
\lim _{n \rightarrow \infty}|r| \frac{\left(\lambda_{n}-\lambda_{n-1}\right)}{\lambda_{n}}=0, \\
\lim _{n \rightarrow \infty}\left|\frac{r\left(\lambda_{n-1}-\lambda_{n-2}\right)+s\left(\lambda_{n}-\lambda_{n-1}\right)}{\lambda_{n}}\right|=0,
\end{gathered}
$$

$$
\begin{aligned}
\lim _{n \rightarrow \infty} \frac{1}{\lambda_{n}} \\
\quad \times \sum_{k=0}^{n-2}\left|r\left(\lambda_{k}-\lambda_{k-1}\right)+s\left(\lambda_{k+1}-\lambda_{k}\right)+t\left(\lambda_{k+2}-\lambda_{k+1}\right)\right|=0 .
\end{aligned}
$$

Now, we have for every $n \geq 1$ that

$$
\begin{aligned}
& \frac{1}{\lambda_{n}} \sum_{k=0}^{n-2}\left|r\left(\lambda_{k}-\lambda_{k-1}\right)+s\left(\lambda_{k+1}-\lambda_{k}\right)+t\left(\lambda_{k+2}-\lambda_{k+1}\right)\right| \\
& \quad=\frac{\lambda_{n-2}}{\lambda_{n}}\left[\frac{1}{\lambda_{n-2}} \sum_{k=0}^{n-2}\left(\lambda_{k}-\lambda_{k-1}\right) z_{k}\right]
\end{aligned}
$$

and since $\lim _{n \rightarrow \infty}\left(\lambda_{n-2} / \lambda_{n}\right)=1$ by (33) and (34), we obtain that by (35)

$$
\lim _{n \rightarrow \infty} \frac{1}{\lambda_{n-2}} \sum_{k=0}^{n-2}\left(\lambda_{k}-\lambda_{k-1}\right) z_{k}=0
$$

which shows that $z=\left(z_{k}\right) \in c_{0}^{\lambda}$ where the sequence $z=\left(z_{k}\right)$ is defined by

$$
z_{k}=\left|\frac{r\left(\lambda_{k}-\lambda_{k-1}\right)+s\left(\lambda_{k+1}-\lambda_{k}\right)+t\left(\lambda_{k+2}-\lambda_{k+1}\right)}{\lambda_{k}-\lambda_{k-1}}\right|
$$

for all $k \in \mathbb{N}$.

Conversely, we suppose that $z=\left(z_{k}\right) \in c_{0}^{\lambda}$. Then we have (37). Further, for every $n \geq 2$, we derive that

$$
\begin{gathered}
\frac{1}{\lambda_{n}} \sum_{k=0}^{n-2}\left|r\left(\lambda_{k}-\lambda_{k-1}\right)+s\left(\lambda_{k+1}-\lambda_{k}\right)+t\left(\lambda_{k+2}-\lambda_{k+1}\right)\right| \\
=\frac{1}{\lambda_{n}} \sum_{k=0}^{n-2}\left(\lambda_{k}-\lambda_{k-1}\right) z_{k} \leq \frac{1}{\lambda_{n-2}} \sum_{k=0}^{n-2}\left(\lambda_{k}-\lambda_{k-1}\right) z_{k} .
\end{gathered}
$$

Then, (37) and (39) together imply that (35) holds. On the other hand, we have for every $n \geq 2$ that

$$
\begin{aligned}
& \left|\frac{r \lambda_{n-2}+s\left(\lambda_{n-1}-\lambda_{0}\right)+t\left(\lambda_{n}-\lambda_{1}\right)}{\lambda_{n}}\right| \\
& \quad=\left|\frac{1}{\lambda_{n}} \sum_{k=0}^{n-2} r\left(\lambda_{k}-\lambda_{k-1}\right)+s\left(\lambda_{k+1}-\lambda_{k}\right)+t\left(\lambda_{k+2}-\lambda_{k+1}\right)\right| \\
& \quad \leq \frac{1}{\lambda_{n}} \sum_{k=0}^{n-2}\left|r\left(\lambda_{k}-\lambda_{k-1}\right)+s\left(\lambda_{k+1}-\lambda_{k}\right)+t\left(\lambda_{k+2}-\lambda_{k+1}\right)\right| .
\end{aligned}
$$

Therefore, it follows by (35)

$$
\lim _{n \rightarrow \infty} \frac{r \lambda_{n-2}+s\left(\lambda_{n-1}-\lambda_{0}\right)+t\left(\lambda_{n}-\lambda_{1}\right)}{\lambda_{n}}=0 .
$$

Particularly if (i) $r=0, s=-1, t=1$, then we obtain $\lim _{n \rightarrow \infty}\left[\left(\lambda_{n}-\lambda_{n-1}\right) / \lambda_{n}\right]=0$, which shows that (33) holds. (ii) $r=-1, s=1, t=0$, and then we obtain $\lim _{n \rightarrow \infty}\left[\left(\lambda_{n-1}-\right.\right.$ $\left.\left.\lambda_{n-2}\right) / \lambda_{n}\right]=0$, which shows that (34) holds. Thus, we deduce by relation (32) that (31) holds. This leads us with Lemma 7 to the consequence that $\widehat{W} \in\left(\ell_{\infty}: c_{0}\right)$. Hence, the inclusion $\ell_{\infty} \subset c_{0}^{\lambda}(\widehat{B})$ holds. Finally, it is obvious that the sequence $y$, defined in the proof of Theorem 6 , is in $c_{0}^{\lambda}(\widehat{B})$ but not in $\ell_{\infty}$, so the inclusion $\ell_{\infty} \subset c_{0}^{\lambda}(\widehat{B})$ is strict.

Theorem 9. The inclusion $\ell_{\infty} \subset \ell_{\infty}^{\lambda}(\widehat{B})$ strictly holds.

Proof. To prove the validity of the inclusion $\ell_{\infty} \subset \ell_{\infty}^{\lambda}(\widehat{B})$, it suffices to show that, for every $x \in \ell_{\infty}^{\lambda}(\widehat{B})$, there exists a positive real number $K$ such that $\|x\|_{\ell_{\infty}^{\lambda}(\widehat{B})} \leq K\|x\|_{\ell_{\infty}}$. Let $x \in \ell_{\infty}$. Then, we have

$$
\begin{aligned}
\|x\|_{\ell_{\infty}^{\lambda}(\widehat{B})} & =\sup _{n \in \mathbb{N}}\left|\frac{1}{\lambda_{n}} \sum_{k=0}^{n}\left(\lambda_{k}-\lambda_{k-1}\right)\left(r x_{k}+s x_{k-1}+t x_{k-2}\right)\right| \\
& \leq \sup _{0 \leq k \leq n}\left|r x_{k}+s x_{k-1}+t x_{k-2}\right| \sup _{n \in \mathbb{N}}\left|\frac{1}{\lambda_{n}} \sum_{k=0}^{n}\left(\lambda_{k}-\lambda_{k-1}\right)\right| \\
& \leq \max \{|r|+|s|+|t|\}\|x\|_{\ell_{\infty}}
\end{aligned}
$$


so that $x \in \ell_{\infty}^{\lambda}(\widehat{B})$ and hence $\ell_{\infty} \subset \ell_{\infty}^{\lambda}(\widehat{B})$. Furthermore, we consider $x=\left(x_{k}\right)$ defined by $x_{k}=\sum_{j=0}^{k} d_{k j}$ for all $k \in \mathbb{N}$. Then we have $\widehat{W} x=e$. Thus, we deduce that $\|x\|_{\ell_{\infty}^{\lambda}(\widehat{B})}=\|\widehat{W} x\|_{\infty}=$ 1. Hence $x=\left(x_{k}\right) \in \ell_{\infty}^{\lambda}(\widehat{B})$.

On the other hand, we know from Theorem 8 that the inclusion $\ell_{\infty} \subset c_{0}^{\lambda}(\widehat{B})$ is strict. Since $c_{0}^{\lambda}(\widehat{B}) \subset \ell_{\infty}^{\lambda}(\widehat{B})$, the inclusion $\ell_{\infty} \subset \ell_{\infty}^{\lambda}(\widehat{B})$ strictly holds.

\section{The $\alpha$-, $\beta$-, and $\gamma$-Duals of the Spaces $\mu^{\lambda}(\widehat{B})$ of Nonabsolute Type}

In this section, we determine the $\alpha$-, $\beta$-, and $\gamma$-duals of the generalized difference sequence spaces $c_{0}^{\lambda}(\widehat{B}), c^{\lambda}(\widehat{B}), \ell_{\infty}^{\lambda}(\widehat{B})$, and $\ell_{p}^{\lambda}(\widehat{B})$ of nonabsolute type.

We will firstly give the definition of $\alpha$-, $\beta$-, and $\gamma$-duals of sequence spaces and secondly we give the lemmas due to [39] which are needed in proving the theorems given in Sections 4 and 5.

For the sequence spaces $\lambda$ and $\mu$, define the set $S(\lambda, \mu)$ by

$$
S(\lambda, \mu)=\left\{z=\left(z_{k}\right) \in w: x z=\left(x_{k} z_{k}\right) \in \mu \forall x \in \lambda\right\} .
$$

With the notation of (43), the $\alpha$-, $\beta$-, and $\gamma$-duals $\lambda^{\alpha}, \lambda^{\beta}$, and $\lambda^{\gamma}$ of a sequence space $\lambda$ are defined by

$$
\lambda^{\alpha}=S\left(\lambda, \ell_{1}\right), \quad \lambda^{\beta}=S(\lambda, c s), \quad \lambda^{\gamma}=S(\lambda, b s) .
$$

Lemma 10. (i) $A \in\left(c_{0}: \ell_{1}\right)=\left(c: \ell_{1}\right)=\left(\ell_{\infty}: \ell_{1}\right)$ if and only if

$$
\sup _{K \in \mathscr{F}} \sum_{n}\left|\sum_{k \in K} a_{n k}\right|<\infty
$$

(ii) $A=\left(a_{n k}\right) \in\left(\ell_{p}: \ell_{1}\right)$ if and only if

$$
\sup _{N \in \mathscr{F}} \sum_{k}\left|\sum_{n \in N} a_{n k}\right|^{q}<\infty .
$$

Lemma 11. $A \in\left(c_{0}: c\right)$ if and only if

$$
\begin{gathered}
\lim _{n \rightarrow \infty} a_{n k}=\alpha_{k}, \quad \text { for each fixed } k \in \mathbb{N}, \\
\sup _{n \in \mathbb{N}} \sum_{k}\left|a_{n k}\right|<\infty .
\end{gathered}
$$

Lemma 12. $A \in(c: c)$ if and only if (47) and (48) hold, and

$$
\lim _{n \rightarrow \infty} \sum_{k} a_{n k} \quad \text { exists. }
$$

Lemma 13. $A \in\left(\ell_{\infty}: c\right)$ if an only if (47) holds and

$$
\lim _{n \rightarrow \infty} \sum_{k}\left|a_{n k}\right|=\sum_{k}\left|\alpha_{k}\right| \text {. }
$$

Lemma 14. $A=\left(a_{n k}\right) \in\left(\ell_{p}: c\right)$ if and only if (47) holds and

$$
\sup _{n \in \mathbb{N}} \sum_{k}\left|a_{n k}\right|^{q}<\infty .
$$

Lemma 15. $A \in\left(c: \ell_{\infty}\right)=\left(c_{0}: \ell_{\infty}\right)=\left(\ell_{\infty}: \ell_{\infty}\right)$ if and only if (48) holds.

Lemma 16. $A=\left(a_{n k}\right) \in\left(\ell_{p}: \ell_{\infty}\right)$ if and only if (51) holds.

Now we consider the following sets:

$$
\begin{aligned}
& f_{1}^{\lambda}=\left\{a=\left(a_{n}\right) \in w: \sup _{K \in \mathscr{F}} \sum_{n}\left|\sum_{k \in K} f_{n k}^{\lambda}\right|<\infty\right\}, \\
& f_{2}^{\lambda}=\left\{a=\left(a_{n}\right) \in w: \sum_{j=k}^{\infty} d_{j k} a_{j} \text { exists for each } k \in \mathbb{N}\right\},
\end{aligned}
$$$$
f_{3}^{\lambda}=\left\{a=\left(a_{n}\right) \in w: \sup _{n \in \mathbb{N}} \sum_{k=0}^{n-1}\left|\widehat{g}_{k}(n)\right|^{q}<\infty\right\},
$$$$
f_{4}^{\lambda}=\left\{a=\left(a_{n}\right) \in w: \sup _{n \in \mathbb{N}}\left|\frac{1}{r} \frac{\lambda_{n}}{\left(\lambda_{n}-\lambda_{n-1}\right)} a_{n}\right|<\infty\right\},
$$$$
f_{5}^{\lambda}=\left\{a=\left(a_{n}\right) \in w: \lim _{n \rightarrow \infty} \sum_{k=0}^{n}\left[\sum_{j=0}^{k} d_{k j}\right] a_{k} \text { exists }\right\},
$$$$
f_{6}^{\lambda}=\left\{a=\left(a_{n}\right) \in w: \sup _{N \in \mathscr{F}} \sum_{k=0}^{\infty}\left|\sum_{n \in N} f_{n k}^{\lambda}\right|^{q}<\infty\right\},
$$$$
f_{7}^{\lambda}=\left\{a=\left(a_{n}\right) \in w: \lim _{n \rightarrow \infty} \sum_{k}\left|v_{n k}^{\lambda}\right|=\sum_{k}\left|\lim _{n \rightarrow \infty} v_{n k}^{\lambda}\right|\right\},
$$

where the matrices $F^{\lambda}=\left(f_{n k}^{\lambda}\right)$ and $V^{\lambda}=\left(v_{n k}^{\lambda}\right)$ are defined as follows:

$$
\begin{gathered}
f_{n k}^{\lambda}= \begin{cases}\left(\frac{d_{n k} \lambda_{k}}{\lambda_{k}-\lambda_{k-1}}-\frac{d_{n, k+1} \lambda_{k}}{\lambda_{k+1}-\lambda_{k}}\right) a_{n}, & (k<n), \\
\frac{1}{r} \frac{\lambda_{n}}{\left(\lambda_{n}-\lambda_{n-1}\right)} a_{n}, & (k=n), \\
0, & (k>n),\end{cases} \\
v_{n k}^{\lambda}= \begin{cases}\widehat{g}_{k}(n), & (k<n), \\
\frac{1}{r} \frac{\lambda_{n}}{\left(\lambda_{n}-\lambda_{n-1}\right)} a_{n}, & (k=n), \\
0, & (k>n)\end{cases}
\end{gathered}
$$


for all $k, n \in \mathbb{N}$ and the $\widehat{g}_{k}(n)$ is defined by

$$
\widehat{g}_{k}(n)=\lambda_{k}\left(\frac{1}{\lambda_{k}-\lambda_{k-1}} \sum_{j=k}^{n} d_{j k} a_{j}-\frac{1}{\lambda_{k+1}-\lambda_{k}} \sum_{j=k+1}^{n} d_{j, k+1} a_{j}\right),
$$

for $k<n$.

Theorem 17. (i) $\left\{c_{0}^{\lambda}(\widehat{B})\right\}^{\alpha}=\left\{c^{\lambda}(\widehat{B})\right\}^{\alpha}=\left\{\ell_{\infty}^{\lambda}(\widehat{B})\right\}^{\alpha}=f_{1}^{\lambda}$.

$$
\text { (ii) }\left\{\ell_{p}^{\lambda}(\widehat{B})\right\}^{\alpha}=f_{6}^{\lambda} \text {. }
$$

Proof. We prove the theorem for the space $c_{0}^{\lambda}(\widehat{B})$. Let $a=$ $\left(a_{n}\right) \in w$. Then, we obtain the equality

$$
a_{n} x_{n}=\sum_{k=0}^{n} d_{n k} \sum_{j=k-1}^{k}(-1)^{k-j} \frac{\lambda_{j}}{\lambda_{k}-\lambda_{k-1}} a_{n} y_{i}=\left(F^{\lambda} y\right)_{n},
$$

$\forall n \in \mathbb{N}$,

by relation (22). Thus we observe by (55) that $a x=\left(a_{n} x_{n}\right) \in$ $\ell_{1}$ whenever $x=\left(x_{k}\right) \in c_{0}^{\lambda}(\widehat{B})$ if and only if $F^{\lambda} y \in \ell_{1}$ whenever $y=\left(y_{k}\right) \in c_{0}$. This means that the sequence $a=\left(a_{n}\right) \in\left\{c_{0}^{\lambda}(\widehat{B})\right\}^{\alpha}$ if and only if $F^{\lambda} \in\left(c_{0}: \ell_{1}\right)$. Therefore we obtain by Lemma 10 with $F^{\lambda}$ instead of $A$ that $a=\left(a_{n}\right) \epsilon$ $\left\{c_{0}^{\lambda}(\widehat{B})\right\}^{\alpha}$ if and only if

$$
\sup _{K \in \mathscr{F}} \sum_{n}\left|\sum_{k \in K} f_{n k}^{\lambda}\right|<\infty
$$

which leads us to the consequence that $\left\{c_{0}^{\lambda}(\widehat{B})\right\}^{\alpha}=f_{1}^{\lambda}$. This completes the proof.

Theorem 18. (i) $\left\{c_{0}^{\lambda}(\widehat{B})\right\}^{\beta}=f_{2}^{\lambda} \cap f_{3}^{\lambda} \cap f_{4}^{\lambda}$ (with $\left.q=1\right)$.

(ii) $\left\{c^{\lambda}(\widehat{B})\right\}^{\beta}=f_{2}^{\lambda} \cap f_{3}^{\lambda} \cap f_{4}^{\lambda} \cap f_{5}^{\lambda}$ (with $q=1$ ).

(iii) $\left\{\ell_{\infty}^{\lambda}(\widehat{B})\right\}^{\beta}=f_{2}^{\lambda} \cap f_{4}^{\lambda} \cap f_{7}^{\lambda}$.

(iv) $\left\{\ell_{p}^{\lambda}(\widehat{B})\right\}^{\beta}=f_{2}^{\lambda} \cap f_{3}^{\lambda} \cap f_{4}^{\lambda}$.

Proof. Consider the equality

$$
\begin{aligned}
\sum_{k=0}^{n} a_{k} x_{k}= & \sum_{k=0}^{n}\left[\sum_{j=0}^{k} d_{k j} \sum_{i=j-1}^{i}(-1)^{j-i} \frac{\lambda_{i}}{\lambda_{j}-\lambda_{j-1}} y_{i}\right] a_{k} \\
= & \sum_{k=0}^{n}\left(\lambda_{k} y_{k}-\lambda_{k-1} y_{k-1}\right)\left[\frac{1}{\lambda_{k}-\lambda_{k-1}} \sum_{j=k}^{n} d_{j k} a_{j}\right] \\
= & \sum_{k=0}^{n-1} \lambda_{k}\left[\frac{\sum_{j=k}^{n} d_{j k} a_{j}}{\lambda_{k}-\lambda_{k-1}}-\frac{\sum_{j=k+1}^{n} d_{j, k+1} a_{j}}{\lambda_{k+1}-\lambda_{k}}\right] y_{k} \\
& +\frac{1}{r} \frac{a_{n} \lambda_{n}}{\left(\lambda_{n}-\lambda_{n-1}\right)} y_{n}
\end{aligned}
$$

$$
\begin{aligned}
& =\sum_{k=0}^{n-1} \widehat{g}_{k}(n) y_{k}+\frac{1}{r} \frac{a_{n} \lambda_{n}}{\left(\lambda_{n}-\lambda_{n-1}\right)} y_{n} \\
& =\left(V^{\lambda} y\right)_{n} ; \quad(n \in \mathbb{N}) .
\end{aligned}
$$

Then we deduce by (57) that $a x=\left(a_{k} x_{k}\right) \in c s$ whenever $x=$ $\left(x_{k}\right) \in c_{0}^{\lambda}(\widehat{B})$ if and only if $V^{\lambda} y \in c$ whenever $y=\left(y_{k}\right) \in c_{0}$. This means that $a=\left(a_{k}\right) \in\left\{c_{0}^{\lambda}(\widehat{B})\right\}^{\beta}$ if and only if $V^{\lambda} \in\left(c_{0}\right.$ : $c)$. Therefore, by using Lemma 11, we obtain

$$
\begin{gathered}
\sum_{j=k}^{\infty} d_{j k} a_{j} \text { exists for each } k \in \mathbb{N}, \\
\sup _{n \in \mathbb{N}} \sum_{k=0}^{n-1}\left|\widehat{g}_{k}(n)\right|<\infty, \\
\sup _{k \in \mathbb{N}}\left|\frac{1}{r} \frac{\lambda_{k}}{\left(\lambda_{k}-\lambda_{k-1}\right)} a_{k}\right|<\infty .
\end{gathered}
$$

Hence, we conclude that $\left\{c_{0}^{\lambda}(\widehat{B})\right\}^{\beta}=f_{2}^{\lambda} \cap f_{3}^{\lambda} \cap f_{4}^{\lambda}$.

Finally, we ended this section with the following theorem which determines the $\gamma$-duals of sequence spaces $c_{0}^{\lambda}(\widehat{B}), c^{\lambda}(\widehat{B}), \ell_{\infty}^{\lambda}(\widehat{B})$, and $\ell_{p}^{\lambda}(\widehat{B})$.

Theorem 19. (i) $\left\{c_{0}^{\lambda}(\widehat{B})\right\}^{\gamma}=\left\{c^{\lambda}(\widehat{B})\right\}^{\gamma}=\left\{\ell_{\infty}^{\lambda}(\widehat{B})\right\}^{\gamma}=f_{3}^{\lambda} \cap$ $f_{4}^{\lambda}$ (with $\left.q=1\right)$.

(ii) $\left\{\ell_{p}^{\lambda}(\widehat{B})\right\}^{\gamma}=f_{3}^{\lambda} \cap f_{4}^{\lambda}$.

\section{Some Matrix Transformations Related to the Spaces $\mu^{\lambda}(\widehat{B})$ of Nonabsolute Type for $\mu \in\left\{c_{0}, c, \ell_{\infty}, \ell_{p}\right\}$}

In this final section, we state some results on the characterization of several classes of matrix mappings on the $c_{0}^{\lambda}(\widehat{B}), c^{\lambda}(\widehat{B}), \ell_{\infty}^{\lambda}(\widehat{B})$, and $\ell_{p}^{\lambda}(\widehat{B})$. We will write throughout for brevity that

$$
\begin{array}{r}
\hat{g}_{n k}(m) \\
=\lambda_{k}\left(\frac{1}{\lambda_{k}-\lambda_{k-1}} \sum_{j=k}^{m} d_{j k} a_{n j}-\frac{1}{\lambda_{k+1}-\lambda_{k}} \sum_{j=k+1}^{m} d_{j, k+1} a_{n j}\right) \\
\quad \text { for } k<m, \\
\widehat{g}_{n k}=\lambda_{k}\left(\frac{1}{\lambda_{k}-\lambda_{k-1}} \sum_{j=k}^{\infty} d_{j k} a_{n j}-\frac{1}{\lambda_{k+1}-\lambda_{k}} \sum_{j=k+1}^{\infty} d_{j, k+1} a_{n j}\right)
\end{array}
$$

for all $k, m, n \in \mathbb{N}$. We assume that the series are convergent in the last equation.

We will begin with lemmas which are needed in the proof of our theorems. 
Lemma 20. The matrix mappings between the BK-spaces are continuous.

Lemma 21. $A=\left(a_{n k}\right) \in\left(c: \ell_{p}\right)$ if and only if

$$
\sup _{F \in \mathscr{F}} \sum_{n}\left|\sum_{k \in F} a_{n k}\right|^{p}<\infty .
$$

Lemma 22. $A=\left(a_{n k}\right) \in\left(c: c_{0}\right)$ if and only if (48) holds and

$$
\begin{gathered}
\lim _{n \rightarrow \infty} a_{n k}=0, \quad \text { for each fixed } k \in \mathbb{N}, \\
\lim _{n \rightarrow \infty} \sum_{k} a_{n k}=0 .
\end{gathered}
$$

Lemma 23. $A \in\left(c_{0}: c_{0}\right)$ if and only if (48) and (61) hold.

Now, we may give our matrix transformations.

Theorem 24. Let $A=\left(a_{n k}\right)$ be an infinite matrix and $1<p<$ $\infty$. Then, $A \in\left(c^{\lambda}(\widehat{B}): \ell_{p}\right)$ if and only if

$$
\sup _{F \in \mathscr{F}} \sum_{n}\left|\sum_{k \in F} \widehat{g}_{n k}\right|^{p}<\infty \text {, }
$$

$\sum_{j=k}^{\infty} d_{j k} a_{n j} \quad$ exists for all fixed $k, n \in \mathbb{N}$,

$$
\sup _{m \in \mathbb{N}} \sum_{k=0}^{m-1}\left|\widehat{g}_{n k}(m)\right|<\infty
$$

$\lim _{k \rightarrow \infty} \frac{1}{r} \frac{\lambda_{k}}{\left(\lambda_{k}-\lambda_{k-1}\right)} a_{n k}=a_{n}$ exists for each fixed $n \in \mathbb{N}$

$$
\begin{gathered}
\left\{\sum_{j=k}^{\infty} d_{j k} a_{n j}\right\} \in c s ; \quad(n \in \mathbb{N}), \\
\left(a_{n}\right) \in \ell_{p} .
\end{gathered}
$$

Proof. If conditions (62)-(67) hold and $x=\left(x_{k}\right)$ is any sequence in the $\operatorname{space} c^{\lambda}(\widehat{B})$ then by using Theorem 18 we have that $\left\{a_{n k}\right\}_{k \in \mathbb{N}} \in\left\{c^{\lambda}(\widehat{B})\right\}^{\beta}$ for all $n \in \mathbb{N}$. Hence, $A$-transform of $x$ exists. Furthermore, since the associated sequence $y=\left(y_{k}\right)$ is in the space $c$, we may write $\lim _{k} y_{k}=l$ for some suitable $l$. Also, the matrix $\widehat{A}=\left(\widehat{g}_{n k}\right)$ is in the class $\left(c: \ell_{p}\right)$ by Lemma 21 and condition (62), where $1<p<\infty$.

Now, we may consider the $m$ th partial sum of the series $\sum_{k} a_{n k} x_{k}$ which is derived by using relation (22):

$$
\begin{array}{r}
\sum_{k=0}^{m} a_{n k} x_{k}=\sum_{k=0}^{m-1} \widehat{g}_{n k}(m) y_{k}+\frac{1}{r} \frac{\lambda_{m}}{\left(\lambda_{m}-\lambda_{m-1}\right)} a_{n m} y_{m} \\
\forall n, m \in \mathbb{N} .
\end{array}
$$

Then, since $y \in c$ and $\widehat{A} \in\left(c: \ell_{p}\right), \widehat{A} y$ exists and so the series $\sum_{k} \widehat{g}_{n k} y_{k}$ converges for every $n \in \mathbb{N}$. Also, it follows by (63) that we have $\lim _{m \rightarrow \infty} \widehat{g}_{n k}(m)=\widehat{g}_{n k}$. Therefore, if we pass to limit in (68) as $m \rightarrow \infty$, then we obtain by (65) that

$$
\sum_{k} a_{n k} x_{k}=\sum_{k} \widehat{g}_{n k} y_{k}+l a_{n}, \quad \forall n \in \mathbb{N}
$$

which can be written as follows:

$$
(A x)_{n}=(\widehat{A} y)_{n}+l a_{n}, \quad \forall n \in \mathbb{N} .
$$

This yields by taking $\ell_{p}$-norm that

$$
\|A x\|_{\ell_{p}} \leq\|\widehat{A} y\|_{\ell_{p}}+|l|\left\|\left(a_{n}\right)\right\|_{\ell_{p}}<\infty .
$$

Consequently, we have that $A x \in \ell_{p}$; that is, $A \in\left(c^{\lambda}(\widehat{B}): \ell_{p}\right)$. Conversely, if $A \in\left(c^{\lambda}(\widehat{B}): \ell_{p}\right)$, we have $\left\{a_{n k}\right\}_{k \in N} \in$ $\left\{c^{\lambda}(\widehat{B})\right\}^{\beta}$ for all $n \in \mathbb{N}$ which implies with Theorem 18 that conditions (63), (64), and (66) are necessary.

On the other hand, since $c^{\lambda}(\widehat{B})$ and $\ell_{p}$ are $B K$-spaces, we have by Lemma 20 that there is a constant $M>0$ such that

$$
\|A x\|_{p} \leq M\|x\|_{\mathcal{C}^{\lambda}(\widehat{B})}
$$

holds for all $x \in c^{\lambda}(\widehat{B})$. Let $F \in \mathscr{F}$. Then, the sequence $z=\sum_{k \in F} b^{(k)}(\lambda)$ is in $c^{\lambda}(\widehat{B})$, where the sequences $b^{(k)}(\lambda)=$ $\left\{b_{n}^{(k)}(\lambda)\right\}_{n \in \mathbb{N}}$ are defined by (27) for every fixed $k \in \mathbb{N}$.

Since $\widehat{W} b^{(k)}(\lambda)=e^{(k)}$ for each fixed $k \in \mathbb{N}$, we have

$$
\|z\|_{\mathcal{C}^{\lambda}(\widehat{B})}=\|\widehat{W}(z)\|_{\ell_{\infty}}=\left\|\sum_{k \in F} \widehat{W} b^{(k)}(\lambda)\right\|_{\ell_{\infty}}=\left\|\sum_{k \in F} e^{(k)}\right\|_{\ell_{\infty}}=1 .
$$

Furthermore, for every $n \in \mathbb{N}$, we obtain by (27) that

$$
A_{n}(z)=\sum_{k \in F} A_{n}\left(b^{(k)}(\lambda)\right)=\sum_{k \in F} \sum_{j} a_{n j} b_{j}^{(k)}(\lambda)=\sum_{k \in F} \widehat{g}_{n k} .
$$

Hence, since inequality (72) is satisfied for the sequence $z \in$ $c^{\lambda}(\widehat{B})$, we have for any $F \in \mathscr{F}$ that

$$
\left(\sum_{n}\left|\sum_{k \in F} \widehat{g}_{n k}\right|^{p}\right)^{1 / p} \leq M
$$

which shows the necessity of (62). Thus, it follows by Lemma 21 that $\widehat{A}=\left(\widehat{g}_{n k}\right) \in\left(c: \ell_{p}\right)$.

Moreover, we consider the sequence $x=\left(x_{k}\right)$ defined by (22) for every $k \in \mathbb{N}$ and suppose that $y=\left(y_{k}\right) \in c \backslash c_{0}$. Then, since $x \in c^{\lambda}(\widehat{B})$ such that $y=\widehat{W} x$ by (18), the transforms $A x$ and $\widehat{A} y$ exist. Hence, the series $\sum_{k} a_{n k} x_{k}$ and $\sum_{k} \widehat{g}_{n k} y_{k}$ are convergent for every $n \in \mathbb{N}$. So we infer that

$$
\lim _{m \rightarrow \infty} \sum_{k=0}^{m-1} \widehat{g}_{n k}(m) y_{k}=\sum \widehat{g}_{n k} y_{k} ; \quad(n \in \mathbb{N}) .
$$

Consequently, we obtain from (68) that

$$
\lim _{m \rightarrow \infty} \frac{1}{r} \frac{\lambda_{m}}{\left(\lambda_{m}-\lambda_{m-1}\right)} a_{n m} y_{m} \quad \text { exists for each fixed } n \in \mathbb{N},
$$


Hence, we deduce that

$$
\lim _{m \rightarrow \infty} \frac{1}{r} \frac{\lambda_{m}}{\left(\lambda_{m}-\lambda_{m-1}\right)} a_{n m} \quad \text { exists for each fixed } n \in \mathbb{N} \text {. }
$$

which leads us to the necessity of (65) and so the relation (68) holds, where $l=\lim _{k} y_{k}$.

Finally, since $A x \in \ell_{p}$ and $\widehat{A} y \in \ell_{p}$, the necessity of (67) is immediate by (68). This completes the proof.

Theorem 25. $A=\left(a_{n k}\right) \in\left(c^{\lambda}(\widehat{B}): \ell_{\infty}\right)$ if and only if (65) and (66) hold, and

$$
\begin{gathered}
\sup _{n \in \mathbb{N}} \sum_{k}\left|\widehat{g}_{n k}\right|<\infty, \\
\left(a_{n}\right) \in \ell_{\infty} .
\end{gathered}
$$

Proof. This is an immediate consequence of Lemma 15 and Theorem 24.

Theorem 26. (i) $A=\left(a_{n k}\right) \in\left(c_{0}^{\lambda}(\widehat{B}): \ell_{p}\right)$ if and only if (62),(63), and (64) hold and

$$
\sup _{k}\left|\frac{1}{r} \frac{\lambda_{k}}{\left(\lambda_{k}-\lambda_{k-1}\right)} a_{n k}\right|<\infty, \quad(n \in \mathbb{N}) .
$$

(ii) $A \in\left(c_{0}^{\lambda}(\widehat{B}): \ell_{\infty}\right)$ if and only if (64), (79), and (81) hold.

Proof. This may be obtained by proceedings as in Theorem 24, above. So, we omit the detail.

Theorem 27. $A=\left(a_{n k}\right) \in\left(c^{\lambda}(\widehat{B}): c\right)$ if and only if (65), (66), and (79) hold and

$$
\lim _{n} a_{n}=a
$$

$$
\begin{gathered}
\lim _{n} \widehat{g}_{n k}=\alpha_{k}, \quad \text { for each fixed } k \in \mathbb{N}, \\
\lim _{n} \sum_{k} \widehat{g}_{n k}=\alpha .
\end{gathered}
$$

Proof. Suppose that $A$ satisfies conditions (65), (66), (79), (82), (83), and (84), and let $x=\left(x_{k}\right)$ be a sequence in the space $c^{\lambda}(\widehat{B})$. Since $(79)$ implies (64), we have by Theorem 18 that $\left\{a_{n k}\right\}_{k \in \mathbb{N}} \in\left\{c^{\lambda}(\widehat{B})\right\}^{\beta}$ for all $n \in \mathbb{N}$. Hence, $A x$ exists. We also observe from (79) and (83) that

$$
\sum_{j=0}^{k}\left|\alpha_{j}\right| \leq \sup _{n \in \mathbb{N}} \sum_{j}\left|\widehat{g}_{n k}\right|<\infty
$$

holds for every $k \in \mathbb{N}$. So $\left(\alpha_{k}\right) \in \ell_{1}$ and hence the series $\sum_{k} \alpha_{k}\left(y_{k}-l\right)$ converges, where $y=\left(y_{k}\right) \in c$ is the sequence connected with $x=\left(x_{k}\right)$ by relation (18) such that $\lim _{k} y_{k}=l$. Also, it is obvious by combining Lemma 12 with conditions (79), (83), and (84) that the matrix $\widehat{A}=\left(\widehat{g}_{n k}\right)$ is in the class $(c: c)$.

Now, by following the similar way used in the proof of Theorem 24, we obtain that relation (69) holds, which can be written as follows:

$$
\sum_{k} a_{n k} x_{k}=\sum_{k} \widehat{g}_{n k}\left(y_{k}-l\right)+l \sum_{k} \widehat{g}_{n k}+l a_{n}, \quad \forall n \in \mathbb{N} .
$$

If we pass the limit in (86) as $n \rightarrow \infty$ we have that

$$
\lim _{n}(A x)_{n}=\sum_{k} \alpha_{k}\left(y_{k}-l\right)+l(\alpha+a)
$$

which shows that $A x \in c$; that is, $A \in\left(c^{\lambda}(\widehat{B}): c\right)$.

Conversely, suppose that $A \in\left(c^{\lambda}(\widehat{B}): c\right)$. Since the inclusion $c \subset \ell_{\infty}$ holds, $A \in\left(c^{\lambda}(\widehat{B}): \ell_{\infty}\right)$. Therefore, the necessity of conditions (65), (66), and (79) is obvious from Theorem 25. Furthermore, consider the sequence $b^{(k)}(\lambda)=$ $\left\{b_{n}^{(k)}(\lambda)\right\}_{n \in \mathbb{N}} \in c^{\lambda}(\widehat{B})$ defined by (27) for every fixed $k \in$ $\mathbb{N}$. Then, one can see that $A b^{(k)}(\lambda)=\left\{\widehat{g}_{n k}\right\}_{n \in \mathbb{N}}$ and hence $\left\{\widehat{g}_{n k}\right\}_{n \in \mathbb{N}} \in c$ for every $k \in \mathbb{N}$ which shows the necessity of (83). Let $z=\sum_{k} b^{(k)}(\lambda)$. Then, since the linear transformation $T: c^{\lambda}(\widehat{B}) \rightarrow c$, defined as in the proof of Theorem 3 by analogy, is continuous and $\widehat{W} b^{(k)}(\lambda)=e^{(k)}$ for each fixed $k \in \mathbb{N}$, we obtain that

$$
(\widehat{W} z)_{n}=\sum_{k}\left(\widehat{W} b^{(k)}(\lambda)\right)_{n}=\sum_{k} \delta_{n k}=1, \quad \text { for each } n \in \mathbb{N}
$$

which shows that $(\widehat{W} z)_{n}=e \in c$ and hence $z \in c^{\lambda}(\widehat{B})$. On the other hand, since $c^{\lambda}(\widehat{B})$ and $c$ are the $B K$-spaces, Lemma 20 implies the continuity of the matrix mapping $A: c^{\lambda}(\widehat{B}) \rightarrow c$. Thus, we have for every $n \in \mathbb{N}$ that

$$
(A z)_{n}=\sum_{k}\left(A b^{(k)}(\lambda)\right)_{n}=\sum_{k} \widehat{g}_{n k} .
$$

This shows the necessity of (84).

Now, it follows by (79), (83), and (84) with Lemma 12 that $\widehat{A}=\left(\widehat{g}_{n k}\right) \in(c: c)$. So by (65), (66) and relation (70) hold for all $x \in c^{\lambda}(\widehat{B})$ and $y \in c$.

Finally, the necessity of (82) is immediate by (70) since $A x \in c$ and $\widehat{A} x \in c$. This completes the proof.

Theorem 28. $A=\left(a_{n k}\right) \in\left(c^{\lambda}(\widehat{B}): c_{0}\right)$ if and only if (65), (66), and (79) hold and

$$
\begin{gathered}
\lim _{n} a_{n}=0, \\
\lim _{n} \widehat{g}_{n k}=0, \quad \text { for each fixed } k \in \mathbb{N}, \\
\lim _{n} \sum_{k} \widehat{g}_{n k}=0 .
\end{gathered}
$$

Proof. This is obtained in the similar way used in the proof of Theorem 27 with Lemma 22 instead of Lemma 12 and so we omit the detail.

Theorem 29. $A=\left(a_{n k}\right) \in\left(c_{0}^{\lambda}(\widehat{B}): c\right)$ if and only if (63), (79), (81), and (83) hold.

Proof. This is an immediate consequence of Lemma 11 and Theorems 18 and 26 (ii).

Theorem 30. $A=\left(a_{n k}\right) \in\left(c_{0}^{\lambda}(\widehat{B}): c_{0}\right)$ if and only if (63), (79), and (83) hold. 
Proof. This is an immediate consequence of Lemma 23 and Theorems 18 and 29.

Theorem 31. $A=\left(a_{n k}\right) \in\left(\ell_{p}^{\lambda}(\widehat{B}): \ell_{\infty}\right)$ if and only if (64) and (81) hold and

$$
\sup _{n \in \mathbb{N}} \sum_{k=0}^{\infty}\left|\widehat{g}_{n k}\right|^{q}<\infty, \quad(n \in \mathbb{N}) .
$$

Proof. Suppose that $A=\left(a_{n k}\right)$ satisfies conditions (64), (81), and (91) and take any $x=\left(x_{k}\right) \in \ell_{p}^{\lambda}(\widehat{B})$. Then, it is obvious that $\left\{a_{n k}\right\}_{k \in \mathbb{N}} \in\left\{\ell_{p}^{\lambda}(\widehat{B})\right\}^{\beta}$ for each $n \in \mathbb{N}$. Hence, $(A x)_{n}=$ $\sum_{k=0}^{\infty} a_{n k} x_{k}$ is convergent for all $x \in \ell_{p}^{\lambda}(\widehat{B})$; that is, $A x$ exists. Also, it is obvious by combining Lemma 16 with condition (91) that $\widehat{A}=\left(\widehat{g}_{n k}\right) \in\left(\ell_{p}: \ell_{\infty}\right)$.

Now, we will show that $A x \in \ell_{\infty}$ for all $x \in \ell_{p}^{\lambda}(\widehat{B})$. By using relation (68) we have that

$$
\sum_{k=0}^{\infty} a_{n k} x_{k}=\sum_{k=0}^{\infty} \widehat{g}_{n k} y_{k}
$$

By applying Hölder's inequality to (92) we obtain that

$$
\left|(A x)_{n}\right|=\left|\sum_{k=0}^{\infty} a_{n k} x_{k}\right| \leq \sum_{k=0}^{\infty}\left|\widehat{g}_{n k}\right|\left|y_{k}\right| \leq\|y\|_{\ell_{p}}\left(\sum_{k=0}^{\infty}\left|\widehat{g}_{n k}\right|^{q}\right)^{1 / q}
$$

which gives us by taking supremum over $n \in \mathbb{N}$ in this last inequality that

$$
\sup _{n \in \mathbb{N}}\left|(A x)_{n}\right| \leq\|y\|_{\ell_{p}} \sup _{n \in \mathbb{N}}\left(\sum_{k=0}^{\infty}\left|\widehat{g}_{n k}\right|^{q}\right)^{1 / q}<\infty
$$

which implies that $A x \in \ell_{\infty}$; that is, $A \in\left(\ell_{p}^{\lambda}(\widehat{B}): \ell_{\infty}\right)$.

Conversely, suppose that $A=\left(a_{n k}\right) \in\left(\ell_{p}^{\lambda}(\widehat{B}): \ell_{\infty}\right)$. Thus, $A x$ exists and bounded for all $x \in \ell_{p}^{\lambda}(\widehat{B})$. Also, $\left\{a_{n k}\right\}_{k \in \mathbb{N}} \in$ $\left\{\ell_{p}^{\lambda}(\widehat{B})\right\}^{\beta}$ for all $n \in \mathbb{N}$ which implies the necessity of (64) and (81). If we define the sequences $\widehat{g}_{n}$ such that $\widehat{g}_{n}=\left\{\widehat{g}_{n k}\right\}_{k \in \mathbb{N}}$ then we have that

$$
\left\|\widehat{g}_{n}\right\|_{\ell_{q}}<\infty
$$

So, bearing in mind (81) one can easily obtain relation (92) by using relation (68). On the other hand, the sequences $a_{n}=$ $\left\{a_{n k}\right\}_{k \in \mathbb{N}}$ define the continuous linear functionals on the space $\ell_{p}^{\lambda}(\widehat{B})$ as follows:

$$
f_{n}(x)=\sum_{k=0}^{\infty} a_{n k} x_{k} ; \quad(n \in \mathbb{N}) .
$$

Since the spaces $\ell_{p}^{\lambda}(\widehat{B})$ and $\ell_{p}^{\lambda}$ are linear isomorphic, we have that

$$
\left\|f_{n}\right\|=\left\|\widehat{g}_{n}\right\|_{\ell_{q}} .
$$

Also, since $\left\{f_{n}(x)\right\}_{n \in \mathbb{N}} \in \ell_{\infty}$, it is obvious that

$$
\sup \left\|f_{n}(x)\right\|<\infty \text {. }
$$

Therefore, by using the Banach-Steinhaus Theorem [38, see pages: 1-2] we obtain that

$$
\sup _{n \in \mathbb{N}}\left\|f_{n}\right\|=\sup _{n \in \mathbb{N}}\left\|\widehat{g}_{n}\right\|_{\ell_{q}}=\sup _{n \in \mathbb{N}}\left(\sum_{k=0}^{\infty}\left|\widehat{g}_{n k}\right|^{q}\right)^{1 / q}<\infty
$$

which shows the necessity of (91). This step completes the proof.

Theorem 32. $A=\left(a_{n k}\right) \in\left(\ell_{p}^{\lambda}(\widehat{B}): c\right)$ if and only if (64), (81), (83), and (91) hold.

Proof. Suppose that conditions (64), (81), (83), and (91) hold. Then, $\left\{a_{n k}\right\}_{k \in \mathbb{N}} \in\left\{\ell_{p}^{\lambda}(\widehat{B})\right\}^{\beta}$ for each $n \in \mathbb{N}$. Hence, the series $\sum_{k=0}^{\infty} a_{n k} x_{k}$ is convergent for each $n \in \mathbb{N}$ and for all $x \in \ell_{p}^{\lambda}(\widehat{B})$ which implies that $A x$ exists. By using (83) we obtain that

$$
\left|\widehat{g}_{n k}\right|^{q} \longrightarrow\left|\alpha_{k}\right|^{q} ; \quad(n \longrightarrow \infty)
$$

Since

$$
\left(\sum_{k=0}^{m}\left|\widehat{g}_{n k}\right|^{q}\right)^{1 / q} \leq \sup _{n \in \mathbb{N}}\left(\sum_{k=0}^{\infty}\left|\widehat{g}_{n k}\right|^{q}\right)^{1 / q}=M
$$

for all $m>0$, in this situation, we see by passing to the limit in the last inequality as $n \rightarrow \infty$ that

$$
\left(\sum_{k=0}^{m}\left|\alpha_{k}\right|^{q}\right)^{1 / q} \leq \sup _{n \in \mathbb{N}}\left(\sum_{k=0}^{\infty}\left|\widehat{g}_{n k}\right|^{q}\right)^{1 / q} .
$$

Because (102) holds for all positive integers $m$, we have that

$$
\left(\sum_{k=0}^{\infty}\left|\alpha_{k}\right|^{q}\right)^{1 / q}<\infty
$$

We remember that $x=\left(x_{k}\right)$ and $y=\left(y_{k}\right)$ are associated sequences by relation (22) where $y=\left(y_{k}\right) \in \ell_{p}$ for $x=\left(x_{k}\right) \in$ $\ell_{p}^{\lambda}(\widehat{B})$.

Let $\varepsilon$ be any positive number. Then, there exists a number $N$ such that

$$
\left(\sum_{k=N}^{\infty}\left|y_{k}\right|^{q}\right)^{1 / q}<\frac{\varepsilon}{4 M}
$$

On the other hand, there is an integer $N_{1}$ such that

$$
\left|\sum_{k=0}^{N}\left\{\widehat{g}_{n k}-\alpha_{k}\right\} y_{k}\right| \leq \frac{\varepsilon}{2}
$$


whenever $n \geq N_{1}$. Therefore, we obtain

$$
\begin{aligned}
& \left|\sum_{k=0}^{\infty}\left\{\widehat{g}_{n k}-\alpha_{k}\right\} y_{k}\right| \\
& \quad \leq\left|\sum_{k=0}^{N}\left\{\widehat{g}_{n k}-\alpha_{k}\right\} y_{k}\right|+\left|\sum_{k=N+1}^{\infty}\left\{\widehat{g}_{n k}-\alpha_{k}\right\} y_{k}\right| \\
& \quad \leq \frac{\varepsilon}{2}+\left(\sum_{k=N+1}^{\infty}\left[\left|\widehat{g}_{n k}\right|+\left|\alpha_{k}\right|\right]^{q}\right)^{1 / q}\left(\sum_{k=N+1}^{\infty}\left|y_{k}\right|^{p}\right)^{1 / p} \\
& \quad \leq \frac{\varepsilon}{2}+2 M \frac{\varepsilon}{4 M}=\varepsilon
\end{aligned}
$$

which shows that

$$
\lim _{n \rightarrow \infty} \sum_{k=0}^{\infty} \widehat{g}_{n k} y_{k}=\sum_{k=0}^{\infty} \alpha_{k} y_{k} .
$$

Since

$$
\lim _{n \rightarrow \infty}(A x)_{n}=\lim _{n \rightarrow \infty} \sum_{k=0}^{\infty} \widehat{g}_{n k} y_{k}=\sum_{k=0}^{\infty} \alpha_{k} y_{k}
$$

we have that $A x \in c$ which implies that $A \in\left(\ell_{p}^{\lambda}(\widehat{B}): c\right)$.

The necessity part can be proved by using the similar way in the proof of Theorem 27, so we omit the detail.

Theorem 33. $A=\left(a_{n k}\right) \in\left(\ell_{p}^{\lambda}(\widehat{B}): c_{0}\right)$ if and only if (64), (81), and (91) hold and

$$
\lim _{n} \widehat{g}_{n k}=0, \quad \text { for each fixed } k \in \mathbb{N} \text {. }
$$

Proof. This result can be proved similarly as the proof of Theorem 32.

\section{Conflict of Interests}

The authors declare that there is no conflict of interests regarding the publication of this paper.

\section{Acknowledgment}

The authors wish to express their deep appreciation to the referees of this paper for their excellent remarks which helped them to present the paper in its final form.

\section{References}

[1] F. Başar, Summability Theory and Its Applications, Bentham Science, Oak Park, Ill, USA, 2011.

[2] C. S. Wang, "On nörlund sequence spaces," Tamkang Journal of Mathematics, vol. 9, no. 2, pp. 269-274, 1978.

[3] P. N. Ng and P. Y. Lee, "Cesàro sequence spaces of non-absolute type," Commentationes Mathematicae. Prace Matematyczne, vol. 20, no. 2, pp. 429-433, 1978.

[4] E. Malkowsky, "Recent results in the theory of matrix transformations in sequence spaces," Matematichki Vesnik, vol. 49, no. 3-4, pp. 187-196, 1997.
[5] B. Altay and F. Başar, "On some Euler sequence spaces of nonabsolute type," Ukrainian Mathematical Journal, vol. 57, no. 1, pp. 3-17, 2005.

[6] E. Malkowsky and E. Savaş, "Matrix transformations between sequence spaces of generalized weighted means," Applied Mathematics and Computation, vol. 147, no. 2, pp. 333-345, 2004.

[7] M. Başarır, "On some new sequence spaces and related matrix transformations," Indian Journal of Pure and Applied Mathematics, vol. 26, no. 10, pp. 1003-1010, 1995.

[8] C. Aydin and F. Başarır, "On the new sequence spaces which include the spaces $c_{0}$ and c," Hokkaido Mathematical Journal, vol. 33, no. 2, pp. 383-398, 2004.

[9] F. Başar, B. Altay, and M. Mursaleen, "Some generalizations of the space $b v_{p}$ of $p$-bounded variation sequences," Nonlinear Analysis: Theory, Methods \& Applications, vol. 68, no. 2, pp. 273287, 2008.

[10] M. Şengönül and F. Başar, "Some new Cesàro sequence spaces of non-absolute type which include the spaces $c_{0}$ and $c$," Soochow Journal of Mathematics, vol. 31, no. 1, pp. 107-119, 2005.

[11] B. Altay, "On the space of $p$-summable difference sequences of order $m$, $(1 \leqq p<\infty)$," Studia Scientiarum Mathematicarum Hungarica, vol. 43, no. 4, pp. 387-402, 2006.

[12] H. Polat and F. Başar, "Some Euler spaces of difference sequences of order m," Acta Mathematica Scientia B, vol. 27, no. 2, pp. 254-266, 2007.

[13] E. Malkowsky, M. Mursaleen, and S. Suantai, "The dual spaces of sets of difference sequences of order $m$ and matrix transformations," Acta Mathematica Sinica, vol. 23, no. 3, pp. 521-532, 2007.

[14] M. Kirişçi and F. Başar, "Some new sequence spaces derived by the domain of generalized difference matrix," Computers \& Mathematics with Applications, vol. 60, no. 5, pp. 1299-1309, 2010.

[15] B. Altay and F. Başar, "Certain topological properties and duals of the domain of a triangle matrix in a sequence space," Journal of Mathematical Analysis and Applications, vol. 336, no. 1, pp. 632-645, 2007.

[16] H. Kizmaz, “On certain sequence spaces," Canadian Mathematical Bulletin, vol. 24, no. 2, pp. 169-176, 1981.

[17] C. Aydın and F. Başar, "Some new difference sequence spaces," Applied Mathematics and Computation, vol. 157, no. 3, pp. 677693, 2004.

[18] C. Aydın and F. Başar, "Some new sequence spaces which include the spaces $l_{p}$ and $1_{\infty}$," Demonstratio Mathematica, vol. 38, no. 3, pp. 641-656, 2005.

[19] C. Aydın and F. Başar, "Some generalizations of the sequence space $a_{b}^{r}$," Iranian Journal of Science and Technology Transaction A: Science, vol. 30, no. 2, pp. 175-190, 2006.

[20] F. Başar and B. Altay, "On the space of sequences of $p$ bounded variation and related matrix mappings," Ukrainian Mathematical Journal, vol. 55, no. 1, pp. 136-147, 2003.

[21] E. Malkowsky and M. Mursaleen, "Some matrix transformations between the difference sequence spaces $\Delta c_{0}(p), \Delta c(p)$ and $\Delta l_{\infty}(p)$, , Filomat, no. 15, pp. 353-363, 2001.

[22] Z. U. Ahmad and M. Mursaleen, "Köthe-Toeplitz duals of some new sequence spaces and their matrix maps," Publications de l'Institut Mathématique, vol. 42, pp. 57-61, 1987.

[23] M. A. Sarı̈öl, “On difference sequence spaces," Journal of Karadeniz Technical University, vol. 10, pp. 63-71, 1987.

[24] Ç. Asma and R. Çolak, "On the Köthe-Toeplitz duals of some generalized sets of difference sequences," Demonstratio Mathematica, vol. 33, no. 4, pp. 797-803, 2000. 
[25] R. Çolak, M. Et, and E. Malkowsky, Some Topics of Sequence Spaces, vol. 63 of Lecture Notes in Mathematics, Firat University Press, Elazı̆̆, Turkey, 2004.

[26] M. Et, "On some difference sequence spaces," Turkish Journal of Mathematics, vol. 17, no. 1, pp. 18-24, 1993.

[27] A. Sönmez and F. Başar, "Generalized difference spaces of nonabsolute type of convergent and null sequences," Abstract and Applied Analysis, vol. 2012, Article ID 435076, 20 pages, 2012.

[28] M. Başarır and E. E. Kara, "On the $\mathrm{m}^{\text {th }}$ order difference sequence space of generalized weighted mean and compact operators," Acta Mathematica Scientia, vol. 33, no. 3, pp. 797-813, 2013.

[29] M. Başarır and E. E. Kara, "On the B-difference sequence space derived by generalized weighted mean and compact operators," Journal of Mathematical Analysis and Applications, vol. 391, no. 1, pp. 67-81, 2012.

[30] E. E. Kara, "Some topological and geometrical properties of new Banach sequence spaces," Journal of Inequalities and Applications, vol. 2013, article 38, 2013.

[31] M. Başarır and E. E. Kara, "On some difference sequence spaces of weighted means and compact operators," Annals of Functional Analysis, vol. 2, no. 2, pp. 114-129, 2011.

[32] E. E. Kara and M. Başarır, "On compact operators and some Euler $B(m)$-difference sequence spaces," Journal of Mathematical Analysis and Applications, vol. 379, no. 2, pp. 499-511, 2011.

[33] M. Candan, "Domain of the double sequential band matrix in the classical sequence spaces," Journal of Inequalities and Applications, vol. 281, no. 1, pp. 1-15, 2012.

[34] M. Candan, "Almost convergence and double sequential band matrix," Acta Mathematica Scientia B, vol. 34, no. 2, pp. 354366, 2014.

[35] M. Mursaleen and A. K. Noman, "On the spaces of $\lambda$-convergent and bounded sequences," Thai Journal of Mathematics, vol. 8, no. 2, pp. 311-329, 2010.

[36] M. Mursaleen and A. K. Noman, "On some new sequence spaces of non-absolute type related to the spaces $l_{p}$ and $l_{\infty} \mathrm{I}$," Filomat, vol. 25, no. 2, pp. 33-51, 2011.

[37] A. Sönmez, "Some new sequence spaces derived by the domain of the triple band matrix," Computers \& Mathematics with Applications, vol. 62, no. 2, pp. 641-650, 2011.

[38] A. Wilansky, Summability through Functional Analysis, vol. 85 of North-Holland Mathematics Studies, North-Holland Publishing, Amsterdam, The Netherlands, 1984.

[39] M. Stieglitz and H. Tietz, "Matrixtransformationen von folgenräumen eine ergebnisübersicht," Mathematische Zeitschrift, vol. 154, no. 1, pp. 1-16, 1977. 


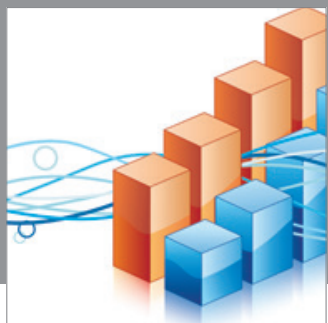

Advances in

Operations Research

mansans

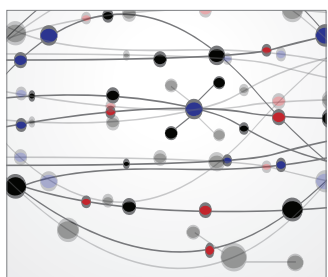

The Scientific World Journal
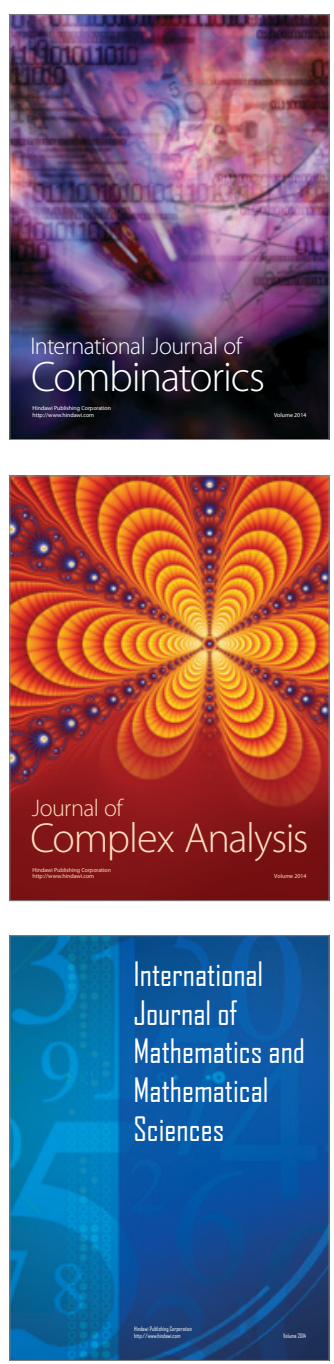
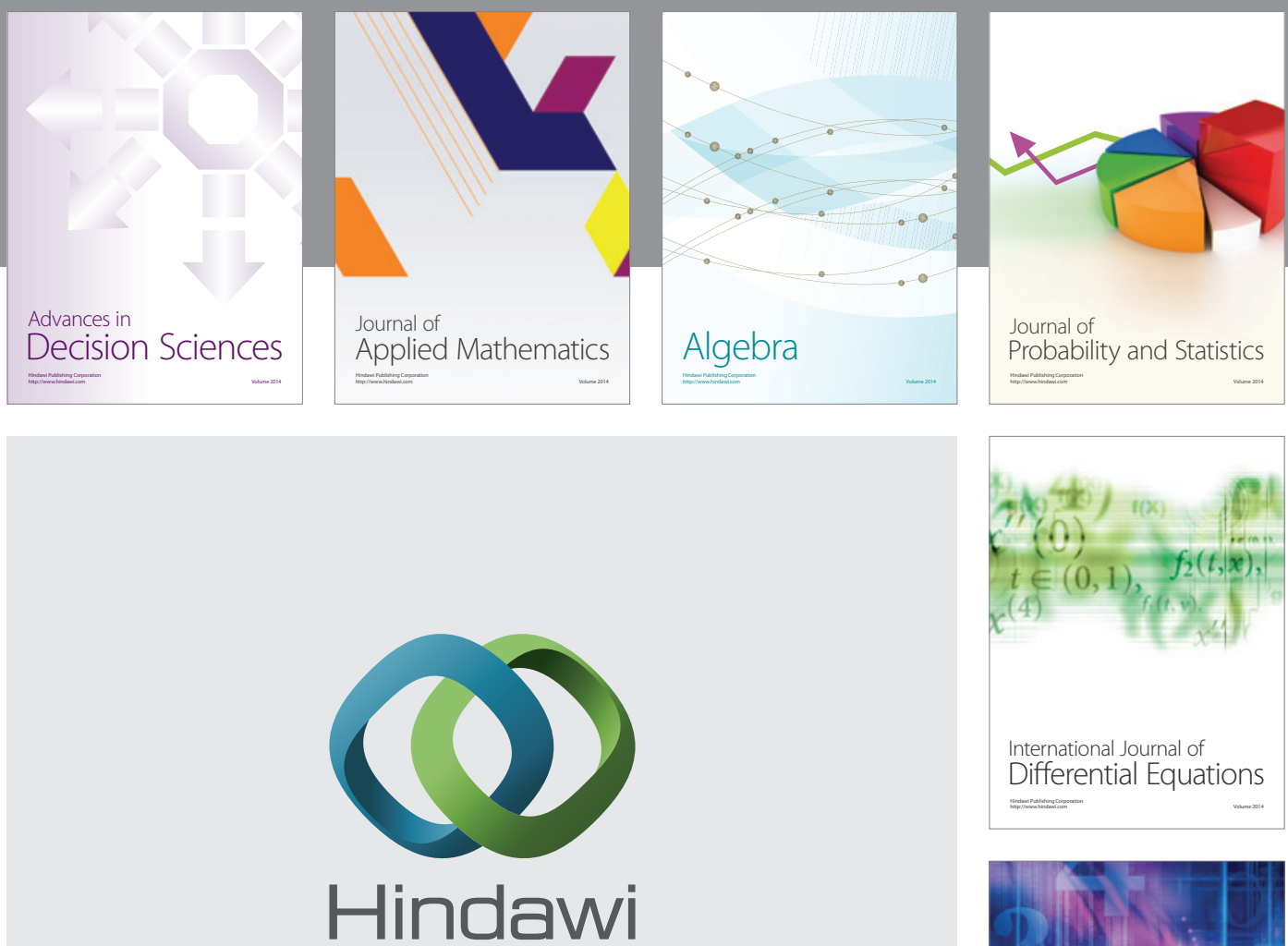

Submit your manuscripts at http://www.hindawi.com
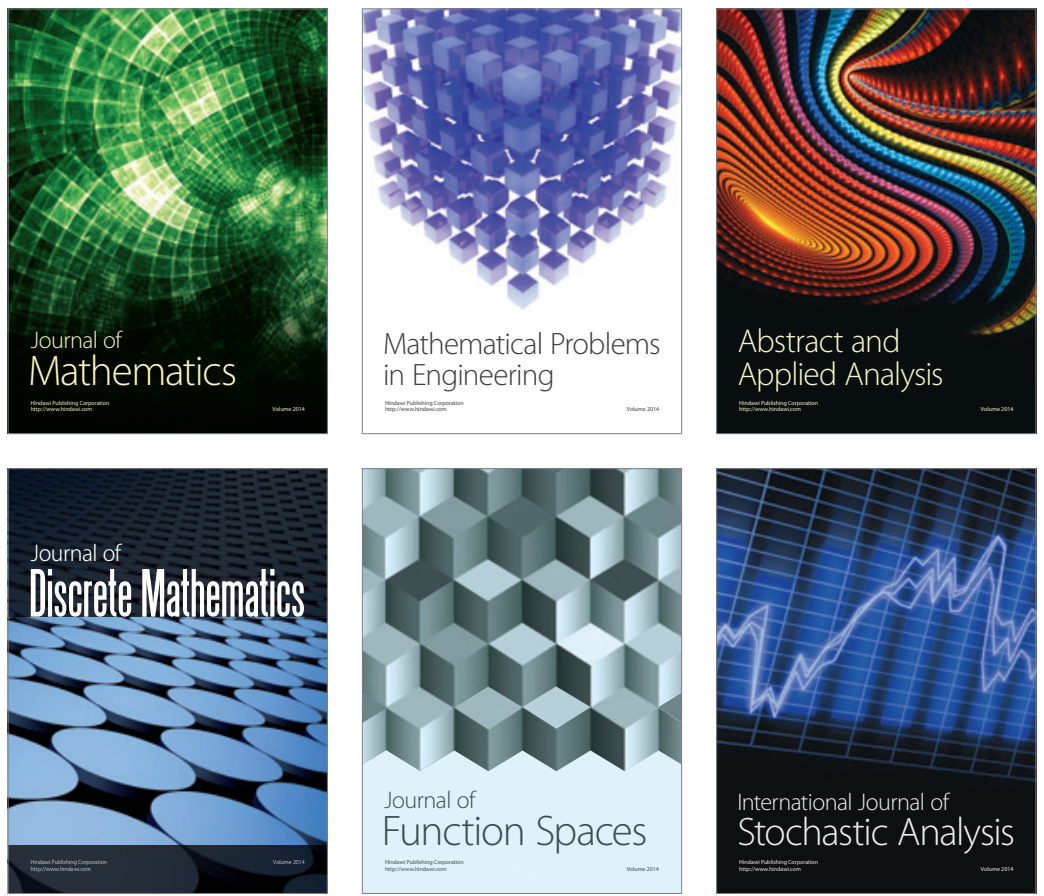

Journal of

Function Spaces

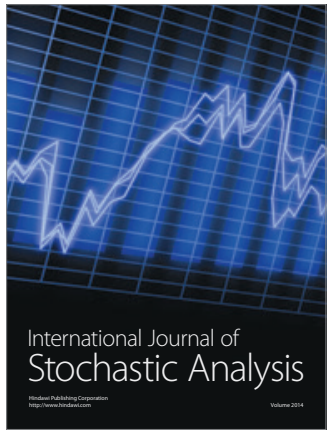

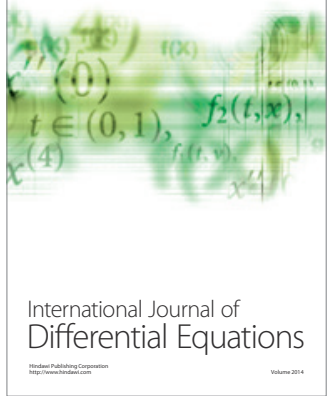
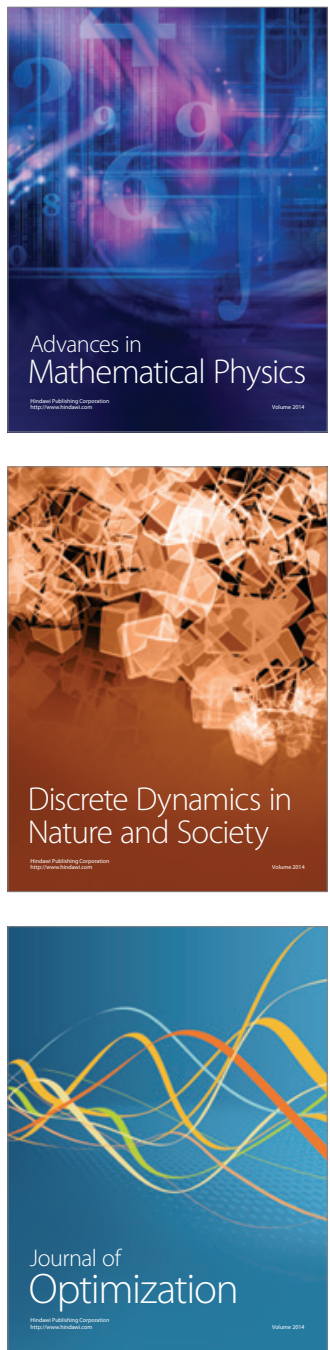